\title{
Formulation of Indomethacin Colon Targeted Delivery Systems Using Polysaccharides as Carriers by Applying Liquisolid Technique
}

\author{
Kadria A. Elkhodairy, ${ }^{1,2}$ Hanna A. Elsaghir, ${ }^{1,3}$ and Amal M. Al-Subayiel ${ }^{1}$ \\ ${ }^{1}$ Department of Pharmaceutics, College of Pharmacy, King Saud University, P.O. Box 22452, Riyadh 11495, Saudi Arabia \\ ${ }^{2}$ Department of Industrial Pharmacy, Faculty of Pharmacy, Alexandria University, Alexandria, Egypt \\ ${ }^{3}$ Department of Pharmaceutics, Faculty of Pharmacy, Cairo University, Cairo, Egypt \\ Correspondence should be addressed to Kadria A. Elkhodairy; elkhodairy53@yahoo.com
}

Received 28 February 2014; Revised 10 April 2014; Accepted 10 April 2014; Published 26 May 2014

Academic Editor: Oluwatoyin A. Odeku

Copyright (C) 2014 Kadria A. Elkhodairy et al. This is an open access article distributed under the Creative Commons Attribution License, which permits unrestricted use, distribution, and reproduction in any medium, provided the original work is properly cited.

\begin{abstract}
The present study aimed at the formulation of matrix tablets for colon-specific drug delivery (CSDD) system of indomethacin (IDM) by applying liquisolid (LS) technique. A CSDD system based on time-dependent polymethacrylates and enzyme degradable polysaccharides was established. Eudragit RL 100 (E-RL 100) was employed as time-dependent polymer, whereas bacterial degradable polysaccharides were presented as LS systems loaded with the drug. Indomethacin-loaded LS systems were prepared using different polysaccharides, namely, guar gum (GG), pectin (PEC), and chitosan (CH), as carriers separately or in mixtures of different ratios of $1: 3,1: 1$, and $3: 1$. Liquisolid systems that displayed promising results concerning drug release rate in both $\mathrm{pH} 1.2$ and $\mathrm{pH} 6.8$ were compressed into tablets after the addition of the calculated amount of E-RL 100 and lubrication with magnesium stearate and talc in the ratio of 1 : 9. It was found that E-RL 100 improved the flowability and compressibility of all LS formulations. The release data revealed that all formulations succeeded to sustain drug release over a period of 24 hours. Stability study indicated that PEC-based LS system as well as its matrix tablets was stable over the period of storage (one year) and could provide a minimum shelf life of two years.
\end{abstract}

\section{Introduction}

Colonic drug delivery has gained increased importance not only for the delivery of the drugs for the treatment of local diseases associated with the colon, such as Chron's diseases, ulcerative colitis, colorectal cancer, and amebiasis, but also for its potential for the delivery of proteins and therapeutic peptides [1].

Natural polysaccharides are now extensively used for the development of solid dosage forms for delivery of drug to the colon. The rationale for the development of a polysaccharide based delivery system for colon is the presence of large amounts of polysaccharides in the human colon as the colon is inhabited by a large number and variety of bacteria which secrete many enzymes, for example, $\beta$-d-glucosidase, $\beta$-dgalactosidase, amylase, pectinase, xylanase, $\beta$-d-xylosidase, and dextranase. A large number of polysaccharides have already been studied for their potential as colon-specific drug carrier systems, such as chitosan, pectin, chondroitin sulphate, cyclodextrin, dextrans, guar gum, inulin, amylose, and locust bean gum [2].

Successful delivery to the colon requires the drug to be in solution form before it arrives at the colon or, alternatively, it should dissolve in the luminal fluids of the colon, but this can be a limiting factor for poorly soluble drugs as the fluid content in the colon is much lower than in the upper part of the gastrointestinal (GI) tract. In addition, because of the high water absorption capacity of the colon, the colonic contents are considerably viscous and their mixing is not efficient; thus, availability of most drugs to the absorptive membrane is low. Furthermore, the stability of the drug is also a concern and must be taken into consideration while 
designing the delivery system. The drug could potentially bind in a nonspecific manner to dietary residues, intestinal secretions, mucus, or fecal matter $[3,4]$.

An important factor that must be considered in designing and formulation of therapeutic dosage form is the aqueous solubility of a drug substance. A drug must possess some aqueous solubility for therapeutic efficacy. For a drug to enter the systemic circulation and exert a therapeutic effect, it must first be in solution. Relatively insoluble compounds often exhibit incomplete or erratic absorption. If the solubility of the drug substance is less than desirable, consideration must be given to improve its solubility and dissolution rate [5]. The methods to accomplish this depend on the chemical nature of the drug and the type of drug product under consideration. Various techniques are reported to improve the dissolution of poorly soluble drugs, including solid dispersions [6], crystal engineering [7], ball milling [8], complexation [9], self-emulsifying drug delivery systems [10], and the use of mesoporous silica carriers [11].

The liquisolid technique [12] has shown promise for improved dissolution rate of poorly water-soluble drugs. The liquisolid technique as described by Spireas [13] is a novel concept, where a liquid may be transformed into a free flowing, readily compressible, and apparently dry powder by simple physical blending with selected carrier and coating material $[13,14]$. The liquid portion, which can be a liquid drug, a drug suspension, or a drug solution in suitable nonvolatile liquid vehicles (liquid medication), is included into the porous carrier material. As the carrier is saturated with liquid, a liquid layer is formed on the particle surface which is instantly adsorbed by the fine coating particles $[15,16]$. The liquisolid compacts are acceptably flowing and compressible powdered forms of liquid medications.

In the liquisolid systems (LSS), even though the drug might be in a solid dosage form, it is held within the powder substrate in solution or in a solubilized, almost molecularly dispersed state $[17,18]$. Therefore, due to their significantly increased wetting properties and surface area of drug available for dissolution, liquisolid compacts of waterinsoluble substances may be expected to display enhanced drug release characteristics and consequently improved oral bioavailability [18-20].

The formulation of poorly water-soluble drugs into different drug delivery systems is usually performed in two steps. The first step is the preformulation study to increase its solubility using an appropriate solubilization technique and hence its oral bioavailability. The second step is the formulation of the required drug delivery system. Thus, the preparation of colon targeted drug delivery system of poorly soluble drug using liquisolid technique could exclude the step of preformulation study and the colon drug delivery system could be prepared directly in one step. The application of liquisolid technique to prepare colon targeted drug delivery system could be considered effective in presenting the drug in a soluble form at the site of absorption, thus overcoming the problem of the low fluid and high viscosity of the colon content.

Indomethacin is a nonsteroidal anti-inflammatory drug (NSAID) used most commonly for the treatment of inflammation and pain resulting from rheumatic arthritis. Indomethacin has anticolorectal cancer activity through cyclooxygenase- (COX-) independent mechanisms [2123]. In spite of its benefits, indomethacin appears to have a high prevalence of gastric side effects [24]. Therefore, indomethacin is considered as a good candidate for colon delivery.

This study aimed at designing and formulation of matrix tablets for colon-specific drug delivery (CSDD) system of IDM by applying liquisolid technique. Liquisolid systems of IDM were prepared using different polysaccharides, namely, GG, PEC, and $\mathrm{CH}$, as carriers separately or in mixtures of different ratios of $1: 3,1: 1$, and $3: 1$. Then a CSDD system based on time-dependent polymethacrylates and enzyme degradable polysaccharides was established. Eudragit RL 100 was employed as time-dependent polymer whereas liquisolid systems loaded with IDM were used as they presented the bacterial degradable polysaccharides. Liquisolid systems that showed promising results concerning drug release rate were compressed into tablets after the addition of the calculated amount of E-RL100 to give IDM matrix tablets for colon delivery.

\section{Experimental}

2.1. Materials. Indomethacin ( $\gamma$-polymorphic form) (IDM) was kindly supplied by Pharco Pharmaceuticals, Alexandria, Egypt, guar gum (GG) by Premcem Gums Ltd., India, pectin (PEC) by BDH Co., England, chitosan (CH) by SigmaAldrich, Germany, microcrystallinecellulose (Avicel pH 102) by FMC Co., USA, colloidal silicone dioxide (Aerosil 200) by FMC Co., Philadelphia, PA, USA, Eudragit RL 100 (ERL100) by Rhone-Pharma, Germany, polyethylene glycol 400 (PEG 400) by European Co. For Pharmaceutical Industries, Egypt, propylene glycol (PG) and polysorbate 80 (Tween 80) by Fisher, United Kingdom, and glycerin by Parchem Fine \& Specialty Chemicals, New York, USA. All other chemicals and buffers were of analytical reagent grades.

2.2. Solubility Studies. To select the best nonvolatile solvent for dissolving or suspending IDM in liquid medication, solubility studies were performed according to the method of Higuchi and Connors [25]. The solubility of IDM was determined in different four nonvolatile solvents, namely, PEG 400, Tween 80, PG, and glycerin. Saturated solutions were prepared by adding excess drug to the vehicles in screwcapped vials. The suspensions were shaken in a thermostatically controlled water bath at $37 \pm 0.5^{\circ} \mathrm{C}$ for 24 hours under constant vibration. The solutions were filtered through a $0.45 \mu \mathrm{m}$ Millipore filter, diluted with phosphate buffer (PB) pH 6.8, and analyzed spectrophotometrically at a wavelength of $318 \mathrm{~nm}$ against blank sample (blank sample contained the same concentration of specific solvent used without drug).

2.3. Calculation of the Loading Factors. In this study, PEG 400 was used as the liquid vehicle, Avicel PH 102 and different polysaccharides, namely, GG, $\mathrm{PEC}$, and $\mathrm{CH}$, as the carriers, 
and Aerosil 200 as the coating material, respectively. The carrier materials were used separately or in mixtures of different ratios $(3: 1,1: 1$, and $1: 3)$. In order to address the flowability and compressibility of LS systems, simultaneously, the "new formulation mathematical model of liquisolid systems" was employed $[12,26]$.

To calculate the loading factor, the liquid medication without drug which is the nonvolatile solvent PEG 400 was added to 20 gram of Avicel PH 102 and blended for $10 \mathrm{~min}$ (reference formulae). The same procedure was followed to calculate the loading factors for different LSS using the different polysaccharides as carriers and mixtures of different carriers in appropriate ratios. The loading factors for each formulation in the appropriate ratios were obtained by using $\mathrm{Lf}=W / Q$ formula, where $W$ is the weight of liquid medication and $Q$ is the amount of the carrier $[12,26]$. The loading factors of eighteen LSS of different composition were determined.

2.4. Preparation of the Liquisolid Systems. The calculated amount of IDM was accurately weighed and dissolved in the determined volume of PEG 400. The resultant liquid medication was added to $20 \mathrm{gm}$ of the carrier in a porcelain dish and blended for $10 \mathrm{~min}$. The appropriate amount of the coating material was then added. Mixing process was carried out in three steps as described by Spireas and Bolton [26]. During the first stage, the system was blended at an appropriate mixing rate of one rotation per second for approximately one minute in order to evenly distribute liquid medication in the powder. In the second stage, the liquid/powder admixture was evenly spread as a uniform layer on the surfaces of mortar and left standing for approximately $5 \mathrm{~min}$ to allow drug solution to be absorbed in the interior of powder particles. In the third stage, powder was spread off the mortar surfaces by means of aluminum spatula. Eighteen LSS of different composition were prepared and evaluated. Only six systems were selected for further studies (Table 1).

\subsection{Evaluation of the Prepared Liquisolid Systems}

2.5.1. Fourier Transform-Infrared (FT-IR) Study. FT-IR spectra of physical mixtures (1:1) of IDM and various excipients, as well as the different LSS, were performed to find out any possible drug-excipients interaction by $\mathrm{KBr}$ disc method using Perkin-Elmer FT-IR series spectrophotometer at a pressure of 90 psi for $5 \mathrm{~min}$ over the range $4000-450 \mathrm{~cm}^{-1}$.

2.5.2. Drug Content. An amount of the prepared LSS (250 mg) was weighed accurately and triturated with $5 \mathrm{~mL}$ of ethanol, and finally the volume was made up to $100 \mathrm{~mL}$ with the $\mathrm{PB}$ pH 6.8. The solution was filtered through a Millipore membrane filter $(0.45 \mu \mathrm{m})$. The filtrate was used to determine the drug content using UV spectrophotometer at $318 \mathrm{~nm}$. Each sample was analyzed in triplicate. Theoretically, each $250 \mathrm{mg}$ of LSS should contain $25 \mathrm{mg}$ of IDM (drug content should be $10 \mathrm{w} / \mathrm{w})$.
2.5.3. Flowability Studies. In order to ensure the flow properties of the LSS that will be selected to be compressed into tablets, angle of repose measurements (fixed height cone method), Carr's index, and Hausner ratio were adopted [27]. In the bulk density measurement, fixed weight of each of the prepared liquisolid powder formulae was placed in graduated cylinder and the volume (V0) occupied was measured and the initial bulk density (D0) was calculated. The graduated cylinder was then tapped at a constant velocity till a constant volume is obtained when the powder is considered to reach the most stable arrangement; the volume of the powder was then recorded as the final bulk volume (Vf), and then the final bulk density (Df) was calculated.

Carr's compressibility index was calculated according to the following equation:

$$
\text { Carr's index } \%=\frac{\mathrm{Df}-\mathrm{D} 0}{\mathrm{Df} \times 100} .
$$

In addition, Hausner ratio was calculated from the following equation:

$$
\text { Hausner's ratio }=\frac{\mathrm{Df}}{\mathrm{D} 0} .
$$

The procedure was done in triplicate. Angle of repose, Carr's compressibility index, and Hausner ratio with the corresponding standard deviations for each of the prepared formulae were calculated (Table 2).

2.5.4. Particle Size Analysis. Particle size of the different LS formulae was determined using the sieving method. A set of sieves $(710-250 \mu \mathrm{m})$ was used. The prepared LSS was placed on the top sieve $(710 \mu \mathrm{m})$ and placed into the sieve shaker and shaking was performed for $10 \mathrm{~min}$. The amount retained on each sieve was weighed. Then the percentage of weight fraction retained on each sieve was calculated. The results were plotted as histograms to show the particle size distribution of each formula under investigation.

2.5.5. In Vitro Dissolution Studies. The dissolution rate of IDM from LS systems was determined using USP XXIV dissolution rate apparatus II at a stirring rate of $100 \pm 2$ and containing $750 \mathrm{~mL}$ of $0.1 \mathrm{~N} \mathrm{HCl}(\mathrm{pH} \mathrm{1.2})$ at $37 \pm 0.5^{\circ} \mathrm{C}$. An amount of each LS formula containing an equivalent of $25 \mathrm{mg}$ IDM filled in a gelatin capsule (size 1) was placed in the dissolution medium. At predetermined time intervals, $5 \mathrm{~mL}$ aliquot was withdrawn and immediately was replaced with an equal volume of dissolution medium at the same temperature. The aliquot withdrawn was filtered through $0.45 \mu \mathrm{m}$ Millipore membrane filter, diluted adequately, and analyzed spectrophotometrically for their IDM content at $\lambda_{\max } 318 \mathrm{~nm}$ against a blank. The same procedure was repeated for each formula using $750 \mathrm{~mL}$ of $\mathrm{PB} \mathrm{pH} 6.8$ for further 4 hours. The experiments were done in triplicate for each of the selected LSS and for control capsule containing $25 \mathrm{mg}$ of IDM.The calculation of dissolution efficiency (DE) has been applied for the evaluation of pharmaceutical equivalence between LSS [28]. 
TABLE 1: Composition of the different liquisolid systems.

\begin{tabular}{lccccc}
\hline Formula code & Carriers & Carriers ratio & $L_{f}$ without IDM & $L_{f}$ with IDM & Drug content \pm SD \\
\hline F1 & GG & - & 0.840 & 0.891 & $18.540 \pm 0.028$ \\
F2 & PEC & - & 0.863 & 0.922 & $19.110 \pm 0.028$ \\
F3 & CH & - & 0.887 & 0.947 & $19.670 \pm 0.037$ \\
F4 & GG + CH & $1: 3$ & 0.884 & 0.945 & $19.390 \pm 0.042$ \\
F5 & PEC + CH & $3: 1$ & 0.884 & 0.945 & $19.110 \pm 0.030$ \\
F6 & PEC + CH & $1: 3$ & 0.863 & 0.922 & $19.670 \pm 0.038$ \\
\hline
\end{tabular}

TABLE 2: Flow characteristics of IDM liquisolid formulations.

\begin{tabular}{lccccc}
\hline Formula's code & Polymer & Ratio of polymers & Angle of repose & Carr's index (\%) & Hauser ratio \\
\hline F1 & GG & - & $34.00 \pm 0.048$ & $19.70 \pm 0.045$ & $1.29 \pm 0.002$ \\
F2 & PEC & - & $36.78 \pm 0.052$ & $26.66 \pm 0.070$ & $1.36 \pm 0.002$ \\
F3 & CH & - & $40.91 \pm 0.063$ & $30.00 \pm 0.057$ & $1.42 \pm 0.004$ \\
F4 & GG + CH & $1: 3$ & $39.90 \pm 0.050$ & $27.43 \pm 0.057$ & $1.39 \pm 0.00$ \\
F5 & PEC + CH & $3: 1$ & $38.09 \pm 0.065$ & $27.50 \pm 0.057$ & $1.38 \pm 0.003$ \\
F6 & PEC + CH & $1: 3$ & $40.70 \pm 0.042$ & $29.17 \pm 0.049$ & $1.41 \pm 0.003$ \\
\hline
\end{tabular}

2.6. Preparation of Compressed Tablets of Liquisolid Systems. The selected LSS were used for the preparation of controlled release colon targeted tablets using E-RL 100.

2.6.1. Fourier Transform-Infrared Spectroscopy (FT-IR) Study. FT-IR spectra of physical mixtures $(1: 1)$ of IDM and various excipients including E-RL 100, as well as the different LS systems and excipients used in preparation of matrix tablet, were performed to find out any possible drug-excipients interaction by $\mathrm{KBr}$ disc method using Perkin-Elmer FT-IR series spectrophotometer.

2.6.2. Flow Characteristics of Tablet Formulations. The flow properties of mixtures of tablet components were studied. Angle of repose, Carr's index, and Hausner ratio were determined as mentioned before. The same procedure was repeated after the addition of the appropriate amount of (Mg.st) : (talc) mixture in the ratio of $1: 9$.

2.6.3. Preparation of IDM Tablets. Mixtures of the prepared LSS and E-RL 100 in the ratio of $2: 1$ were prepared by adding the calculated amount of E-RL 100 geometrically to each of the selected LS systems, namely, F2, F3, F4, F5, and F6, and then mixed thoroughly for 10 minutes. A weighed amount of the lubricant ( $3 \% \mathrm{w} / \mathrm{w}$ of the LSS-E-RL mixture) was then added and mixed thoroughly for another 10 minutes. Then the powder mixture was compressed into tablet using a single punch tablet press with $12 \mathrm{~mm}$ punch and die a pressure of $10-12 \mathrm{Kg} / \mathrm{cm}^{2}$. The tablet weight was varied from 435 to $450 \mathrm{mg}$ depending on the drug content of each system. Each tablet was prepared to contain an amount of LS system equivalent to $25 \mathrm{mg}$ of IDM. Therefore, two tablets of each formulation were used in the dissolution study (Table 4). Indomethacin conventional compressed tablets were prepared by direct compression of the physical mixtures of the drug, carrier, coat, E-RL 100, and lubricant. The conventional tablets were prepared as reference formulae for comparison (Table 5).

\subsection{Evaluation of the Prepared Tablets}

2.7.1. Drug Content. IDM content in different LS tablet formulations was determined by accurately weighing 10 tablets of each formula individually. Each tablet was then crushed and dissolved in $100 \mathrm{~mL} \mathrm{~PB} \mathrm{pH} \mathrm{6.8,} \mathrm{and} \mathrm{then} \mathrm{the}$ solution was filtered, properly diluted, and then measured spectrophotometrically at $\lambda_{\max }$ of $318 \mathrm{~nm}$; thereafter, IDM content of each tablet was determined.

2.7.2. Morphological Examination of Tablet. A tablet was placed in a Petri dish and immersed in $75 \mathrm{~mL} 0.1 \mathrm{~N} \mathrm{HCl}$ solution ( $\mathrm{pH}$ 1.2). The liquid was removed carefully after 2 hours with a syringe and a photograph of the tablet was taken with a digital camera fitted with zoom lens (NIKON COOLPIX L18, Japan). The same tablet was again immersed in $75 \mathrm{~mL}$ of $\mathrm{PB} \mathrm{pH}$ 6.8. Liquid was then removed after 2 hours and a photograph of the tablet was taken. Tablet diameter was measured and the average of three measurements was considered. Further study was conducted using F2 matrix tablet to emphasize the swelling of the matrix along the vertical axis. The matrix tablet was placed in a beaker containing $35 \mathrm{~mL}$ of dissolution medium. The test was carried out in $\mathrm{pH} 1.2$ for 2 hours then the matrix was transferred to $\mathrm{pH} 6.8$ and the swelling behavior was recorded for 24 hours.

2.7.3. In Vitro Dissolution Rate. Dissolution rate of IDM from LS matrix tablets was determined using USP XXIV dissolution rate apparatus I (Pharma Test, Germany) at a stirring rate of $100 \pm 2$ and containing $750 \mathrm{~mL}$ of $0.1 \mathrm{~N} \mathrm{HCl}$ ( $\mathrm{pH} 1.2$ ) at $37 \pm 0.5^{\circ} \mathrm{C}$ for 2 hours then the medium was rendered alkaline to $\mathrm{pH} 6.8$ by the addition of the calculated amount of dihydrogen sodium phosphate. The dissolution 
studies at $\mathrm{pH} 6.8$ were conducted for further 24 hours. Two tablets of each formula, containing an equivalent of $50 \mathrm{mg}$ IDM, were placed in the dissolution medium. At predetermined time intervals, aliquot of $5 \mathrm{~mL}$ from the dissolution medium was withdrawn and immediately replaced with an equal volume of preheated fresh dissolution medium at $37 \pm 0.5^{\circ} \mathrm{C}$. The aliquots withdrawn were filtered through $0.45 \mu \mathrm{m}$ Millipore membrane filter, adequately diluted, and analyzed spectrophotometrically for their IDM content at $\lambda_{\max } 318 \mathrm{~nm}$ using UV-Spectrophotometer (Genesys TM 5, Thermospectronic, USA). The experiments were done in triplicate for each of the selected LSS. The mean percentage cumulative amount of the drug dissolved was plotted against time.

2.7.4. Preparation of Mimic Enzymatic Media of the Colon (Rat Cecal Matter (RCM)). A group of 5 male Albino Wister rats each weighing 150-200 g and maintained on normal diet (soaked grain of polysaccharide) was used to induce enzymes specifically acting on each polysaccharide used. The rats were then killed using $\mathrm{CO}_{2}$ asphyxiation, 45 minutes before the study. The abdomen was opened and the cecal was traced, legated at both ends, dissected, and immediately transferred into $\mathrm{PB} \mathrm{pH}$ 6.8, previously bubbled with $\mathrm{CO}_{2}$. The cecal bags were opened; their contents individually were weighed, pooled, and then suspended in $\mathrm{pH} 6.8$ to give $2 \% \mathrm{w} / \mathrm{v}$ dilution. As the cecum is naturally anaerobic, all operations were carried out under $\mathrm{CO}_{2}$ [29]. The procedure was performed in accordance with the guidelines of the local institutional animal ethics committee.

2.7.5. In Vitro Drug Release in Rat Cecal Matter. Drug release studies in the presence of rat cecal content were also carried out using USP dissolution test apparatus I but with slight modification. After completing the test in $\mathrm{pH} 1.2$ for 2 hours and $\mathrm{pH} 7.4$ for 3 hours, basket containing tablets was immersed in $250 \mathrm{~mL}$ beaker containing $\mathrm{PB}$ solution $(\mathrm{pH}$ 6.8) and rat cecal content was maintained in the jars of the dissolution apparatus for up to 24 hours. Samples of $5 \mathrm{~mL}$ each were withdrawn at different time intervals $(6,7$, 8,9 , and 24 hours), filtered using filter paper, and assayed spectrophotometrically for IDM at $318 \mathrm{~nm}$. The same volume of fresh medium bubbled with $\mathrm{CO}_{2}$ was added after each withdrawn sample [30].

2.8. Drug Release Kinetics. In order to investigate the mechanism of drug release from compressed tablets, the release data were fitted into first-order, zero-order, Higuchi models, and the Korsmeyer model [31].

2.9. Stability on Storage. Storage test was performed on LSS selected for preparation of compressed tablets. Liquisolid systems and their corresponding matrix tablet formulations were kept on shelf for 9-12 months (to study the effect of fluctuation in temperature and humidity on the proposed formulations). The different temperatures and relative humidity $(\mathrm{RH})$ under which this study was carried out were in the range of $25-45^{\circ} \mathrm{C}$ and $10-16 \%$. These conditions are those of area where the formulations were prepared and would be stored and sold. All formulations were tested for any changes in physical appearance, drug content, in vitro release profiles, and any chemical interactions by FT-IR analysis.

2.10. Statistical Analysis. All the results were expressed as mean values \pm standard deviation (SD). One way analysis of variance (ANOVA) with Tukey's multiple comparisons post hoc (Graph-Pad prism 6 program) was used to test for significance, at a 5\% significance level. Statistical difference dealing $(P<0.05)$ was considered significant.

\section{Results and Discussion}

3.1. Solubility Studies. Solubility studies revealed that the solubility of IDM can be improved in presence of PEG 400. It was found that solubility of IDM increased to $95.53 \mathrm{mg} / \mathrm{mL}$ in PEG 400. A higher fraction of the drug in PEG 400 is in the molecular state and this would help to increase dissolution rate of the drug because the drug is already dissolved [32].

3.2. Determination of the Loading Factor. Composition of different LSS and their loading factors are summarized in Table 1. The loading factors varied from 0.840 (F1) to 0.887 (F3), respectively, in absence of the drug. However, upon the addition of IDM, the loading factors increased because the weight of liquid medication increased. It has been reported that, in conventional LS formulation, it is difficult to prepare formulation with good flowability and compatibility when loading factor is above 0.25 [12]. In this study, as the prepared LSS showed loading factors above 0.25 , they were considered unacceptable formulations with respect to flowability and compatibility.

\subsection{Evaluation of the Prepared Liquisolid Systems}

3.3.1. Fourier Transform-Infrared Spectroscopy (FT-IR) Study. The FT-IR spectra of selected formulations were determined and only one spectrum of a representative formulation is included. FT-IR spectrum of PEC-based LS system as well as PEC-based physical mixture is presented in Figure 1. The characteristic peaks of the pure drug were compared with peaks obtained from the respective LSS (F2). It was observed that characteristic peaks of IDM appear with identical or with minor differences, at frequencies of 3370.33 and $1717.14 \mathrm{~cm}^{-1}$ corresponding to carboxylic $\mathrm{O}-\mathrm{H}$ and $\mathrm{C}=\mathrm{O}$ stretch, respectively. The spectrum shows also characteristic peaks at $2961.65 \mathrm{~cm}^{-1}(\mathrm{C}-\mathrm{H}$ stretching vibrations), $1691.83 \mathrm{~cm}^{-1}$ ( $\mathrm{C}=\mathrm{O}$ stretching vibrations), $1234.15 \mathrm{~cm}^{-1}$ (asymmetric aromatic O-C stretching), $1086.55 \mathrm{~cm}^{-1}$ (symmetric aromatic $\mathrm{O}-\mathrm{H}$ stretching), and $1479.50 \mathrm{~cm}^{-1}$ (C-C stretching) [33].

FT-IR spectrum of PEC shows characteristic peaks at $3421.68 \mathrm{~cm}^{-1}$ and $2932 \mathrm{~cm}^{-1}$, which is due to the presence of $-\mathrm{OH}$ and $-\mathrm{CH}$ stretching vibration peaks, respectively. The peaks observed at $1735.19 \mathrm{~cm}^{-1}$ can be assigned to carboxymethyl (-COOCH3) peaks of PEC. The peaks at 

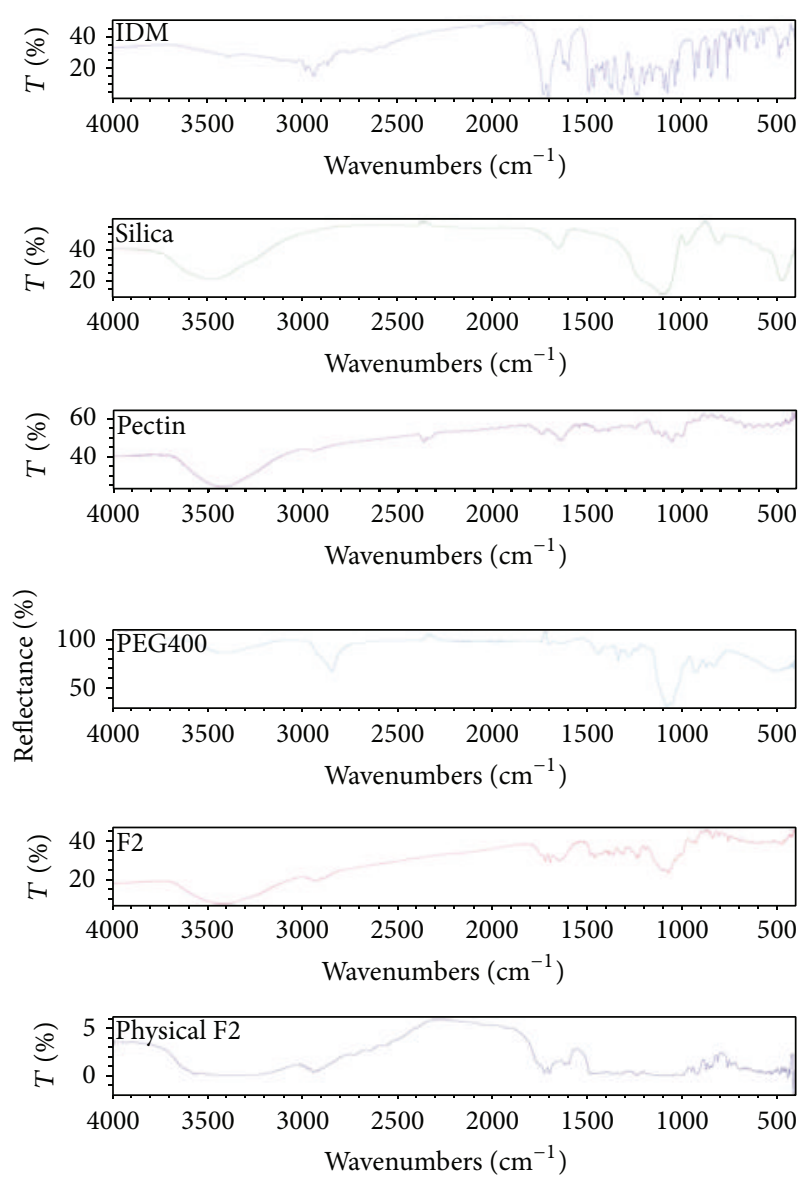

FIGURE 1: FTIR spectra of IDM, PEC, silica, PEG 400, liquisolid system, and physical mixture of formulation F2.

1437.66 and $1357 \mathrm{~cm}^{-1}$ could be assigned to $\mathrm{CH}_{2}$ and $\mathrm{OH}$ bending vibration peaks, respectively. The spectrum shows $\mathrm{O}-\mathrm{H}$ bending at $1052.59 \mathrm{~cm}^{-1}, \mathrm{C}=\mathrm{O}$ stretching at $1637.03 \mathrm{~cm}^{-1}$, and $\mathrm{C}-\mathrm{H}$ stretching at $2361.07 \mathrm{~cm}^{-1}$ [34]. It can be seen that the peaks of the LSS F2 as well as F2 physical mixture are the sum of the characteristic peaks of the drug and the corresponding excipients used. The spectra of Figure 1 indicated the compatibility between the drug and each excipient used for preparation of LSS F2. The same results were obtained with the other prepared LSS (F1, F3, F4, F5, and F6) indicating no drug excipient interactions.

3.3.2. Drug Content. The results of the drug content studies revealed that the amount of the drug present in $250 \mathrm{mg}$ LSS varied from $18.54 \pm 0.028 \mathrm{mg}(7.42 \%)$ for F1 to $19.67 \pm$ $0.037 \mathrm{mg}$ (7.87\%) for F3.

3.3.3. Flow Properties. Liquisolid systems F1, F2, F4, F5, and F6 showed range of angle of repose; the lowest value was 34.00 (F1) and the highest value was 40.91 (F3). These values indicated that these formulations were LSS with good to fair flowability according to the USP [35]. Liquisolid systems F3 and F6 showed angles of repose of $40.91^{\circ}$ and $40.70^{\circ}$, thus, exhibiting passable flow characteristics [35]. In addition,

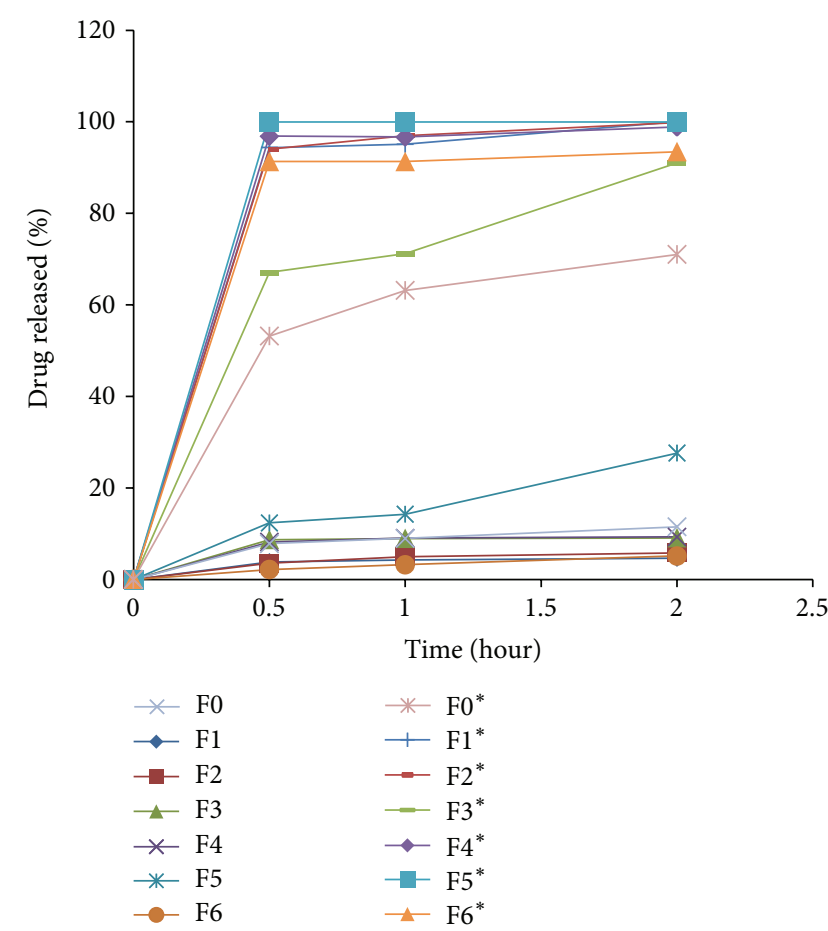

FIGURE 2: Release profiles of IDM from different liquisolid formulations (F1-F6) and pure drug (F0) in $0.1 \mathrm{~N} \mathrm{HCl} \mathrm{(F)} \mathrm{and} \mathrm{in} \mathrm{PB}$ pH 6.8 $\left(\mathrm{F}^{*}\right)$.

Hausner ratios and Carr's index of LS systems F2-F6 were higher than 1.250 and $21.00 \%$, respectively, indicating fair flow characteristics [27]. Formulation F1 (GG-based LS) had Hausner ratio and Carr's index of $19.70 \%$ and 1.29 , respectively, indicating good flow properties (Table 2).

3.3.4. Particle Size Analysis. In general, the highest percentage of the weight fraction of the particles was found in the particle size range of $250-500 \mu \mathrm{m}$. The lowest percentage of weight fraction of the coarser particles was found in the particle size range of $500-600 \mu \mathrm{m}$ except for F3 that showed high\% of weight fraction in this range. Formulation F4 showed nearly equal percentage of weight fraction of the particles in the ranges of $250-500$ and $600-710 \mu \mathrm{m}$. Depending on the results of particle size analysis, average particle size of $375 \mu \mathrm{m}$ was used for further evaluation.

3.3.5. In Vitro Dissolution Studies. Dissolution profiles of IDM LSS contained in hard gelatin capsules and pure IDM containing capsule are illustrated in Figure 2. In general, all formulations showed a decrease in drug release rate in $0.1 \mathrm{~N}$ $\mathrm{HCl}$ and an increase in drug release rate in $\mathrm{PB} \mathrm{pH}$ 6.8. This general observation may be attributed to the low solubility of acidic drug (pKa 4.5 ) in $0.1 \mathrm{~N} \mathrm{HCl}$ and its high solubility in the basic medium ( $\mathrm{pH}$ 6.8). Release characteristics are summarized in Table 3 which includes the rate of drug dissolution from all formulations after $30 \mathrm{~min}$ (DR $30 \mathrm{~min}$ ) and its corresponding percentage of dissolution efficiency (DE\% $30 \mathrm{~min}$ ) in both acidic and alkaline mediums. Release 
TABLE 3: Dissolution parameters of different formulations of IDM liquisolid compact.

\begin{tabular}{|c|c|c|c|c|}
\hline Formula's code & $\mathrm{DR}_{30 \min }(\mathrm{pH} 1.2)$ & $\% \mathrm{DE}_{30 \min }(\mathrm{pH} 1.2)$ & $\mathrm{DR}_{30 \min }(\mathrm{pH} 6.8)$ & $\% \mathrm{DE}_{30 \min }(\mathrm{pH} 6.8)$ \\
\hline $\mathrm{F}^{*}{ }^{*}$ & 7.84 & 16.99 & 53.19 & 18.72 \\
\hline $\mathrm{F} 1$ & 3.23 & 12.77 & 94.00 & 23.58 \\
\hline $\mathrm{F} 2$ & 3.53 & 15.13 & 94.55 & 23.66 \\
\hline F3 & 8.70 & 22.68 & 67.10 & 20.07 \\
\hline $\mathrm{F} 4$ & 7.65 & 20.62 & 73.88 & 22.99 \\
\hline F5 & 4.86 & 21.35 & 80.50 & 22.64 \\
\hline F6 & 7.42 & 19.54 & 73.75 & 19.52 \\
\hline
\end{tabular}

TABLE 4: Composition of IDM tablets prepared using liquisolid systems and E-RL.

\begin{tabular}{lccccc}
\hline Formula's code & Carrier & Amount $(\mathrm{mg})$ & Mg st.: talc & Total weight of tablet \\
& & LS* system & E-RL & $1: 9$ \\
\hline F2 & PEC & 327.05 & 109.02 & 13.08 & 449.14 \\
F3 & CH & 317.74 & 105.91 & 12.71 & 436.36 \\
F4 & GG + CH (3:1) & 322.33 & 107.44 & 12.89 & 442.66 \\
F5 & PEC + CH (3:1) & 327.05 & 109.02 & 13.08 & 449.14 \\
F6 & PEC + CH (1:3) & 317.74 & 105.91 & 12.71 & 436.36 \\
\hline
\end{tabular}

LS$^{*}$ system: liquisolid system equivalent to $25 \mathrm{mg}$ of IDM.

profiles of IDM from all formulations F1-F6 in $0.1 \mathrm{~N} \mathrm{HCl}$ and $\mathrm{PB}$ pH 6.8 are illustrated in Figure 3. It is obvious that $\mathrm{CH}$-based LSS (F3) showed the highest drug release rate with $\mathrm{DE} \%$ of 22.68 after 30 min of dissolution experiment in acid medium, whereas PEC-based LSS (F2) exhibited lower drug release rate in acidic medium with $\mathrm{DE} \%$ of 15.13 , after the same time of dissolution test in $\mathrm{pH}$ 1.2. Guar gum-based LS system (F1) exhibited the least drug release rate in $\mathrm{pH} 1.2$ with DE\% of 12.77 after 30 min of the dissolution test. When LSS of GG came into contact with the dissolution medium, they took up water and swelled, forming gelled particles. Then the dissolved drug diffuses out of the swollen, gelled GG [36, 37].

It has been suggested that gel formation is caused by hydrogen bonding between free carboxyl groups on the PEC molecules and also between the hydroxyl groups of neighboring molecules [38]. At low $\mathrm{pH}$, ionization of the carboxylate groups is suppressed and this results in a reduction in hydration of the carboxylic acid groups and reduced number of negative charges. This decrease in the number of negative charges not only lowers the attraction between PEC and water molecules but also lowers the repulsive forces between PEC molecules themselves. Therefore, polysaccharide molecules no longer repel each other over their entire length, and, as a result, they can associate and form a gel which reduces drug release [38].

Chitosan, being basic with $\mathrm{pKa}$ of $6.2-7$, dissolves in acidic $\mathrm{pH}$ showing higher drug release in $0.1 \mathrm{~N} \mathrm{HCl}$. Therefore, F3 (CH-based LSS) showed higher drug release rate than $\mathrm{F} 1$ and $\mathrm{F} 2$ after 2 hours of dissolution in $\mathrm{pH} 1.2$ [39].

In $\mathrm{PB} \mathrm{pH}$ 6.8, it was observed that the drug release rate depended on the extent of polymer hydration, swelling, gelling, and erosion. Guar gum gives $\mathrm{pH}$-independent drug release due to its nonionic nature. It is not affected by ionic strength or $\mathrm{pH}$ [40]. As a result, high drug release rate in PB pH 6.8 from GG-LSS (F1) was observed. An amount of $94.00 \%$ of drug content was released with a DE\% of 23.58 after 30 min of dissolution experiment in buffer $\mathrm{pH}$ 6.8. In case of $\mathrm{PEC}$, in $\mathrm{pH}$ 6.8, most of the unesterified carboxyl groups are ionized producing a negative charge on the molecule which, together with the hydroxyl groups, causes it to attract layers of water. The repulsive forces between these groups, due to their negative charge, can be sufficiently strong to prevent the formation of a gel PEC network [38]. As a result, an increase in drug release rate was noticed from PEC-based LSS of IDM (F2) with a DE\% of 23.66 after 30 min of dissolution test in $\mathrm{pH}$ 6.8. Chitosan, in $\mathrm{pH}$ 6.8, underwent hydration, swelling, and gelling which was followed by slow erosion and reduction in drug release from its LS system (F3). It can be seen that an amount of $67.10 \%$ of drug content was released with a DE\% of 20.07 after $30 \mathrm{~min}$ of dissolution test in $\mathrm{pH} 6.8$.

The effect of mixtures of different hydrophilic polysaccharides upon IDM release from the LSS was also studied. Drug release rate from LSS containing mixtures of GG and $\mathrm{CH}$ in different ratios $(1: 1,3: 1$, and $1: 3)$ was studied. Guar gum and $\mathrm{CH}$ mixture in the ratio of $1: 1$ showed increased drug release rate in acidic and alkaline medium compared to drug release from F1 or F3 LSS alone. On the other hand, GG : $\mathrm{CH}$ mixture in the ratio of $3: 1$ LSS showed the highest retardation of drug release rate (the results are not included). Formulation F4 (GG : CH mixture in the ratio of 1:3) was selected for further study as it showed a reasonable drug release rate retardation depending on the extent of hydration, swelling, and gelling of each hydrophilic polymer.

On using PEC and $\mathrm{CH}$ as a mixture for LS system (Figure 2 and Table 3), it can be seen that the percentage of drug release from LSS F5 (containing the two polymers in 
TABLE 5: Composition of compressed tablets of IDM using physical mixtures.

\begin{tabular}{|c|c|c|c|c|c|c|c|c|}
\hline \multirow[b]{2}{*}{ Formula code } & \multirow[b]{2}{*}{ Carrier } & \multicolumn{6}{|c|}{ Amount (mg) } & \multirow[b]{2}{*}{ Total weight of tablet } \\
\hline & & Coat & GG & PEC & $\mathrm{CH}$ & E-RL & $\begin{array}{c}\text { Mg st : talc } \\
1: 9\end{array}$ & \\
\hline $\mathrm{F} 2$ & PEC & 14.38 & - & 287.67 & - & 109.02 & 13.08 & 449.14 \\
\hline F3 & $\mathrm{CH}$ & 13.94 & & & 278.80 & 105.91 & 12.71 & 436.36 \\
\hline $\mathrm{F} 4$ & $\mathrm{GG}+\mathrm{CH}(1: 3)$ & 14.16 & 70.79 & & 212.37 & 107.44 & 12.89 & 442.66 \\
\hline F5 & $\mathrm{PEC}+\mathrm{CH}(3: 1)$ & 14.38 & & 226.53 & 75.510 & 109.02 & 13.08 & 449.14 \\
\hline F6 & $\mathrm{PEC}+\mathrm{CH}(1: 3)$ & 13.94 & & 69.70 & 209.10 & 105.91 & 12.71 & 436.36 \\
\hline
\end{tabular}
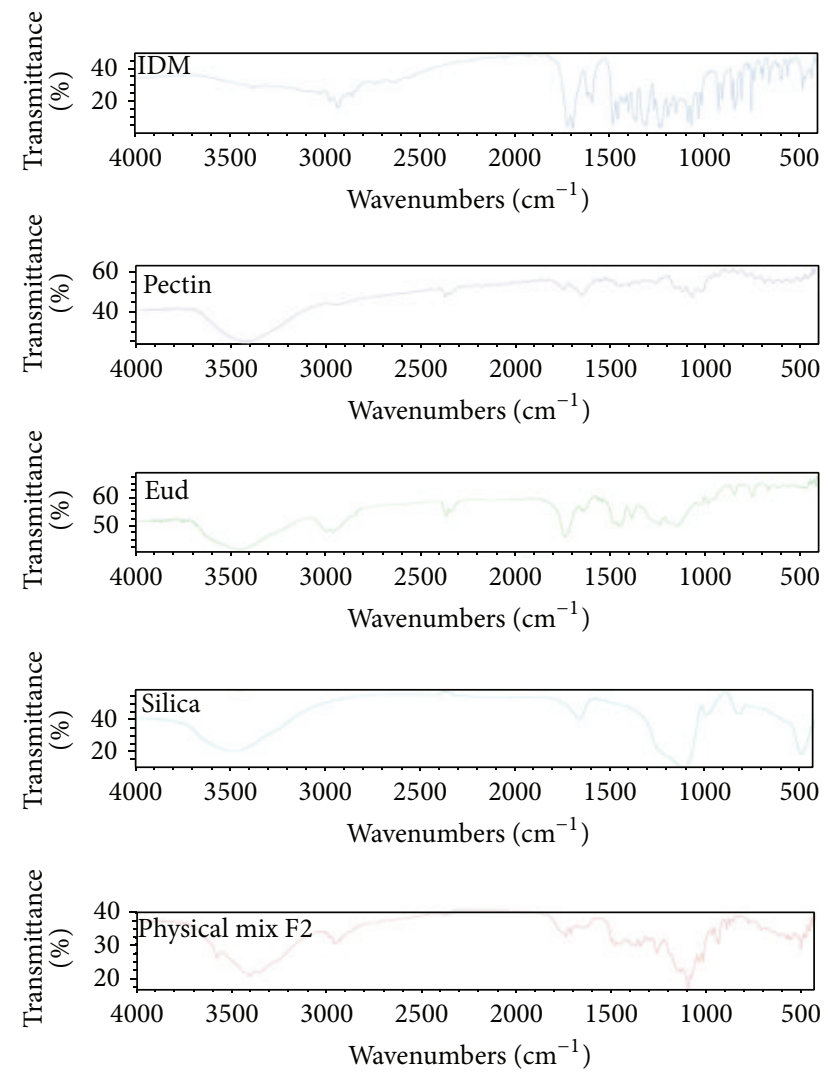

(a)
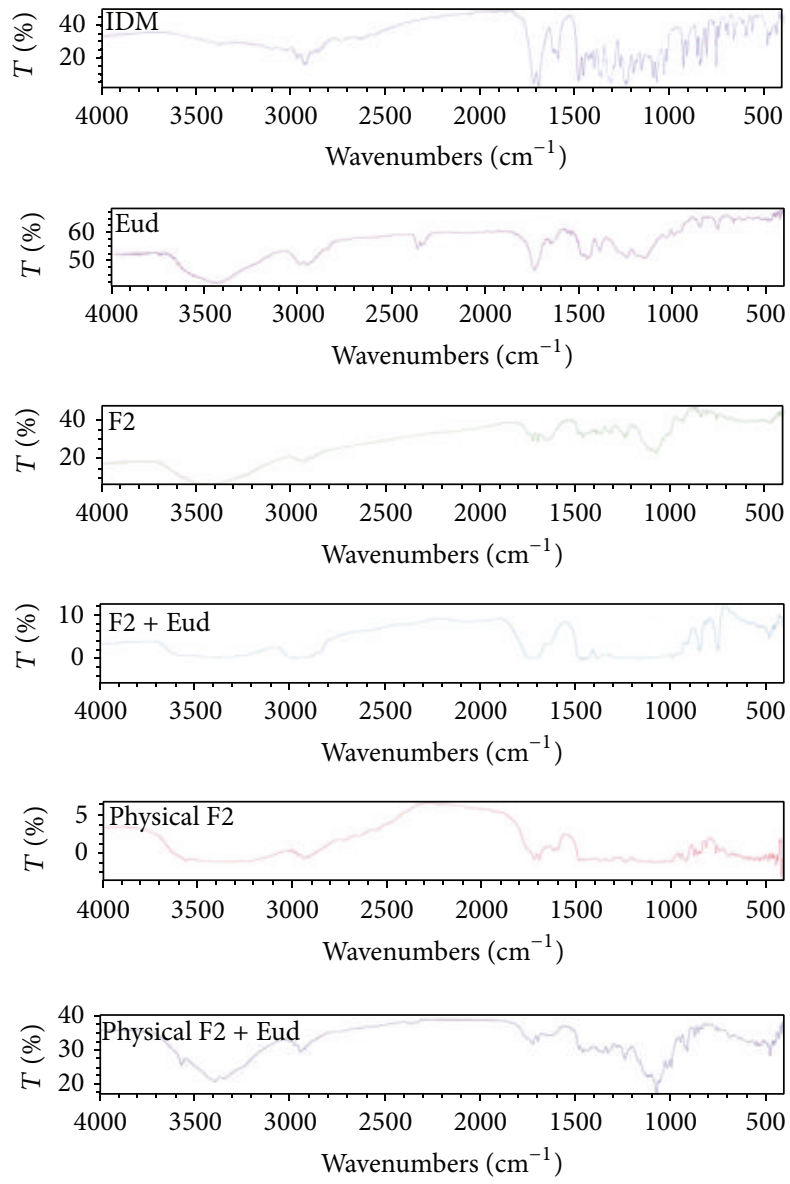

(b)

FIGURE 3: (a) IR spectra of IDM, PEC, silica, E-RL 100, and physical mixture formulation F2. (b) IR spectra of IDM, E-RL 100, formulation F2 LS, formulation F2 LS mixed with E-RL 100, formulation F2 PM, and liquisolid formulation F2 mixed with E-RL.

the ratio of $1: 1$ ) was higher than F2 (containing PEC alone) and lower than $\mathrm{F} 3$ (containing $\mathrm{CH}$ alone) in $\mathrm{pH}$ 1.2. This can be explained on the basis that $\mathrm{CH}$ promotes the drug release in acidic medium due to its dissolution in acidic $\mathrm{pH}$. On the other hand, in $\mathrm{PB}$ pH 6.8, ionization of carboxyl groups increased the repulsion force due to their negative charges, thus preventing the formation of a PEC network resulting in an increase in drug release rate. Increasing the ratio of PEC in formulation (F6) by 3 -fold reduced drug release rate in both $\mathrm{pHs}$. The increased ratio of PEC resulted in the formation of more dense gel upon hydration which extended time to erode and dissolve.
3.3.6. Consideration for Selection of E-RL 100. Different approaches have been studied for targeting drugs to the colon. The main systems are $\mathrm{pH}$-based, time-dependent, and bacterially degradable. However, due to variations in physiological conditions of patients, one system alone could not be completely reliable on colonic drug delivery. Therefore, researches have been notably performed with a combination of the aforementioned systems.

Most of the commercially available systems for colonspecific drug delivery utilize Eudragit polymers (i.e., L100 and S100), soluble at $\mathrm{pH} \mathrm{7,} \mathrm{or} \mathrm{cellulose} \mathrm{acetate} \mathrm{phthalate,}$ dissolving at $\mathrm{pH} 6$ as enteric materials. Eudragit RL and 
RS polymers have been proposed in various studies for colon targeting because of their ammonium groups content allowing a low solubility in gastric fluids [41, 42].

Eudragit RL 100 is cationic copolymer of methacrylate with quaternary ammonium groups. It is inert resins, insoluble at physiologic $\mathrm{pHs}$ and have $\mathrm{pH}$-independent swelling properties. It is compressible and erodible and due to the presence of $10 \%$ quaternary ammonium group the Eudragit matrix is permeable [43].

Eudragit RS (E-RS) 100 and Eudragit RL (E-RL) 100 were used to produce microcarriers for a specific colon release of budesonide for the treatment of inflammatory bowel diseases [44].

The objective of another study was to produce and evaluate a novel colon-specific drug delivery system of 5aminosalicylic acid matrix pellets using a mixture of Eudragit RS and Eudragit RL as time-dependent polymers and pectin as a bacterially degradable polysaccharide [45].

\subsubsection{Consideration for Selection of Liquisolid Systems.} Liquisolid system containing GG (F1) showed the lower drug release rate compared to the other systems as well as the pure drug. Preliminary trials revealed that GG formed dense and thick gel that decreased the drug release from the compressed matrix. Therefore, F4 containing mixture of GG and $\mathrm{CH}$ in the ratio of $1: 3$ was selected to modulate drug release rate from pure GG formulation (F1). Formulation F2 containing PEC was selected since PEC is anionic polysaccharide, insoluble in acidic medium but soluble in alkaline medium. Chitosan containing LS system (F3) was selected to test the efficacy of E-RL in inhibiting the drug release in $0.1 \mathrm{~N} \mathrm{HCl}$, since $\mathrm{CH}$ is cationic polymer that is soluble in acidic medium. F5 and F6 containing mixture of $\mathrm{PEC}$ and $\mathrm{CH}$ in the ratios of $1: 3$ and $3: 1$, respectively, were also selected as they could modulate the drug release rate from pure $\mathrm{PEC}$ formulation (F2) and pure $\mathrm{CH}$ formulation (F3).

The obtained results indicated that all the prepared LSS had poor flow characteristics which limited its formulation into tablet dosage form unless other materials could be incorporated to improve the flow properties. In addition, due to the high solubility and swelling properties of polysaccharides in aqueous media, their dosage forms are unable to prevent the release of drugs during their transit through the stomach and the small intestine.

Therefore, a number of studies have been conducted to use a combination of polymethacrylates and polysaccharides in order to prepare more suitable dosage forms for targeting drugs to the colon [45-48]. Based on the above, each of the selected LS formulations was mixed with the appropriate amounts of E-RL 100 and lubricant and then compressed into tablets.

\section{Precompression Evaluation}

4.1. Fourier Transform-Infrared Spectroscopy (FT-IR) Study. The pure drug (IDM) and the solid admixture of drug and various excipients used in the preparation of matrix tablets were characterized by FT-IR spectroscopy to test their compatibility. The spectra of PEC-based formulations were included as examples. FT-IR spectra of IDM and its physical mixtures (PM) with PEC, silica, and E-RL 100 are demonstrated in Figure 3(a). All characteristic peaks of IDM are present in their original position denoting the absence of any possible interaction between IDM and E-RL or pectin. The characteristic peaks of IDM at $3370.33 \mathrm{~cm}^{-1}$ and $1717.14 \mathrm{~cm}^{-1}$ corresponding to carboxylic $\mathrm{O}-\mathrm{H}$ and $\mathrm{C}=\mathrm{O}$ stretch, respectively, were detected in the PM. It was also observed that the intensity of the peaks in FT-IR spectra of PM containing IDM and excipients was slightly reduced which might be due to the adsorption of the drug on more or less amorphous excipients. In general, it can be noticed that the FT-IR spectra of PM containing IDM, PEC, E-RL, and silica are superimposed with the characteristic peak of IDM. Figure 3(b) illustrates the FT-IR spectra of PEC, E-RL, PECbased LSS loaded with IDM (F2), and mixture of F2 and E-RL used for tableting. It was noticed that the peaks presented the sum of the peaks of IDM and PEC in F2 formulations. FT-IR spectra of the mixture of F2 and E-RL show, in addition to the F2 formulation' peaks, the characteristic peaks of E-RL. Thus, it can be concluded that there was no interaction between the content of LSS F2 and E-RL.

4.2. Flow Characteristics of Tablet Formulations. Although the selected LSS for preparation of tablets exhibited the lower drug dissolution rate in $\mathrm{pH} 1.2$, yet they showed bad flow properties. In general, addition of E-RL 100 to the liquisolid systems of IDM improved its flow characteristics; also, addition of Mg.st: talc in the ratio of $1: 9$ as lubricant resulted in a further improvement in flow characteristics of the selected formulations (Table 6).

\section{Postcompression Evaluation}

5.1. Drug Content of Matrix Tablets. It was observed that all the prepared tablets complied with the test of drug content uniformity according to the USP, in which each individual content was between $85 \%$ and $115 \%$ of the average content.

5.2. Morphological Examination of Matrix Tablet. Visual observation indicated that the tablet matrices appeared to swell and a viscous gel mass was created when they came into contact with the medium. The degree of swelling with the formation of continuous and homogenous gel layer around the matrix was related to the hydration of each biodegradable polymer or combination of polymers in different pHs. Morphological examination offormulation F2 was only included (Figure 4). In the case of hydration of PEC matrix tablets (F2) in acidic medium ( $\mathrm{pH} 1.2)$, the outer hydrated surface layer formed around the tablets could be seen visually to possess a very different consistency from that of the tablets hydrated in alkaline medium ( $\mathrm{pH} 6.8$ ). The hydrated layer (in acidic medium) was not viscous and adhesive in nature but represented a tough and more or less regularly swelled surface (Figure 4(a)). This is probably due to the fact that $\mathrm{PEC}$ is rapidly converted to pectinic acid, at $\mathrm{pH}$ 
TABLE 6: Flow properties of mixtures of tablet components.

\begin{tabular}{|c|c|c|c|c|c|}
\hline Formula's code & Polymer & Ratio of polymers & Angle of repose & Carr's index (\%) & Hauser ratio \\
\hline $\bar{F} 2^{*}$ & PEC & - & $36.78 \pm 0.015$ & $26.66 \pm 0.016$ & $1.36 \pm 0.016$ \\
\hline $\mathrm{F} 2+\mathrm{E}-\mathrm{RL}$ & PEC & - & $27.83 \pm 0.009$ & $21.46 \pm 0.012$ & $1.27 \pm 0.013$ \\
\hline $\mathrm{F} 2+\mathrm{E}-\mathrm{RL}+\mathrm{Mg}$ st $:$ talc $1: 9$ & PEC & - & $26.32 \pm 0.017$ & $21.09 \pm 0.011$ & $1.25 \pm 0.011$ \\
\hline $\mathrm{F} 2+\mathrm{E}-\mathrm{RL}(\mathrm{PM})^{* *}$ & PEC & - & $25.02 \pm 0.022$ & $19.78 \pm 0.012$ & $1.13 \pm 0.011$ \\
\hline $\mathrm{F}^{*}$ & $\mathrm{CH}$ & - & $40.91 \pm 0.019$ & $29.90 \pm 0.008$ & $1.42 \pm 0.008$ \\
\hline $\mathrm{F} 3+\mathrm{E}-\mathrm{RL}$ & $\mathrm{CH}$ & - & $35.00 \pm 0.021$ & $21.20 \pm 0.016$ & $1.37 \pm 0.016$ \\
\hline $\mathrm{F} 3+\mathrm{E}-\mathrm{RL}+\mathrm{Mg}$ st $:$ talc $1: 9$ & $\mathrm{CH}$ & - & $33.10 \pm 0.014$ & $20.83 \pm 0.021$ & $1.32 \pm 0.021$ \\
\hline $\mathrm{F} 3+\mathrm{E}-\mathrm{RL}(\mathrm{PM})^{* *}$ & $\mathrm{CH}$ & - & $30.20 \pm 0.025$ & $20.84 \pm 0.020$ & $1.26 \pm 0.020$ \\
\hline $\mathrm{F} 4^{*}$ & $\mathrm{GG}+\mathrm{CH}$ & $1: 3$ & $39.90 \pm 0.009$ & $31.67 \pm 0.010$ & $1.46 \pm 0.011$ \\
\hline $\mathrm{F} 4+\mathrm{E}-\mathrm{RL}$ & $\mathrm{GG}+\mathrm{CH}$ & $1: 3$ & $32.00 \pm 0.022$ & $21.47 \pm 0.006$ & $1.27 \pm 0.007$ \\
\hline $\mathrm{F} 4+\mathrm{E}-\mathrm{RL}+\mathrm{Mg}$ st $:$ talc $1: 9$ & $\mathrm{GG}+\mathrm{CH}$ & $1: 3$ & $29.45 \pm 0.014$ & $21.00 \pm 0.013$ & $1.28 \pm 0.012$ \\
\hline $\mathrm{F} 4+\mathrm{E}-\mathrm{RL}(\mathrm{PM})^{* *}$ & $\mathrm{GG}+\mathrm{CH}$ & $1: 3$ & $26.00 \pm 0.021$ & $17.17 \pm 0.021$ & $1.20 \pm 0.021$ \\
\hline $\mathrm{F}^{*}$ & $\mathrm{PEC}+\mathrm{CH}$ & $3: 1$ & $38.09 \pm 0.019$ & $27.50 \pm 0.011$ & $1.38 \pm 0.011$ \\
\hline $\mathrm{F} 5+\mathrm{E}-\mathrm{RL}$ & $\mathrm{PEC}+\mathrm{CH}$ & $3: 1$ & $30.46 \pm 0.100$ & $21.46 \pm 0.070$ & $1.27 \pm 0.070$ \\
\hline $\mathrm{F} 5+\mathrm{E}-\mathrm{RL}+\mathrm{Mg}$ st $:$ talc $1: 9$ & $\mathrm{PEC}+\mathrm{CH}$ & $3: 1$ & $28.99 \pm 0.022$ & $20.32 \pm 0.009$ & $1.24 \pm 0.009$ \\
\hline $\mathrm{F} 14+\mathrm{E}-\mathrm{RL}(\mathrm{PM})^{* *}$ & $\mathrm{PEC}+\mathrm{CH}$ & $3: 1$ & $28.50 \pm 0.120$ & $18.77 \pm 0.013$ & $1.21 \pm 0.012$ \\
\hline $\mathrm{F}^{*}$ & $\mathrm{PEC}+\mathrm{CH}$ & $1: 3$ & $40.70 \pm 0.021$ & $29.00 \pm 0.011$ & $1.41 \pm 0.011$ \\
\hline $\mathrm{F} 6+\mathrm{E}-\mathrm{RL}$ & $\mathrm{PEC}+\mathrm{CH}$ & $1: 3$ & $33.90 \pm 0.017$ & $21.56 \pm 0.013$ & $1.20 \pm 0.013$ \\
\hline $\mathrm{F} 6+\mathrm{E}-\mathrm{RL}+\mathrm{Mg}$ st $:$ talc $1: 9$ & $\mathrm{PEC}+\mathrm{CH}$ & $1: 3$ & $30.45 \pm 0.110$ & $20.87 \pm 0.011$ & $1.25 \pm 0.011$ \\
\hline $\mathrm{F} 6+\mathrm{E}-\mathrm{RL}(\mathrm{PM})^{* *}$ & $\mathrm{PEC}+\mathrm{CH}$ & $1: 3$ & $29.46 \pm 0.009$ & $21.72 \pm 0.010$ & $1.18 \pm 0.010$ \\
\hline
\end{tabular}

$\mathrm{F}^{*}$ : liquisolid system.

$\mathrm{PM}^{* *}$ : physical mixture.

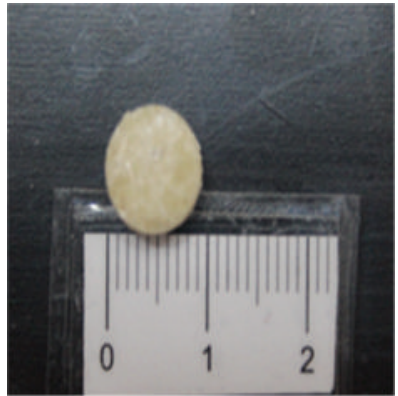

Zero time

(a)

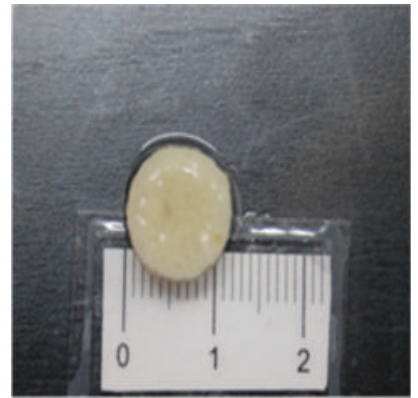

After $2 \mathrm{hr}$ in $\mathrm{pH} 1.2$

(b)

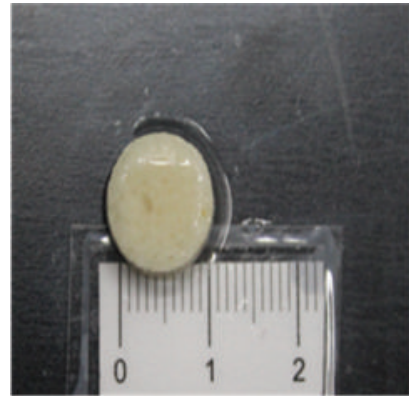

After $2 \mathrm{hr}$ in $\mathrm{pH} 6.8$

(c)

FIGURE 4: Matrix tablet of IDM-PEC liquisolid system (F2).

1-2, which has the ability to swell on hydration being virtually insoluble but it has lower swelling capacity compared to PEC [47]. In addition, it has been proved that gelation of high methoxylated PEC usually occurs at $\mathrm{pH}<3.5$ and its swelling was related to the formation of hydrogen bonds between the hydrophobic groups of polysaccharide chains [47]. Based on the above, PEC in acidic medium swells forming insoluble gel which reduced drug release rate in gastric environment.

Slight increase in tablet diameter was noticed which may be due to the swelling of both E-RL and pectinic acid. Swelling of E-RL did not form homogenous gel layer around the matrix tablet. In general, no disintegration of tablet matrices was observed indicating that the adhesive force existing between the components of the tablets was high enough to prevent tablets disintegration in both acidic and alkaline media. These results are not in agreement with the work done by
Ofokansi and Kenechukwu [48]. They concluded that E-RL 100 exerted significant effect on the force of adhesion of the tablet ingredients, thereby increasing the disintegration time.

Figure 5 shows the swelling behavior of F2 matrix tablet in $\mathrm{pH} 1.2$ for 2 hours and in $\mathrm{pH} 6.8$ for 24 hours. It is obvious that, once the matrix was placed in the dissolution medium, swelling of high methoxylated PEC occurred which was manifested by the white gel network on the tablet. By time, it was observed that the gel formed did not form a continuous layer around the matrix tablet. The gel network entrapped the drug and prevented its release in $0.1 \mathrm{~N} \mathrm{HCl}$. Eudragit polymer present on the surface did not swell as the quaternary ammonium groups reacted with the chloride ions of the dissolution medium [46]. In pH 6.8, almost all the tablet matrices appeared to be covered with continuous layer of gel due to hydration and swelling of PEC and E-RL. Erosion 


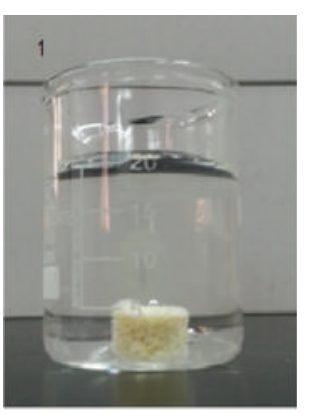

(1)

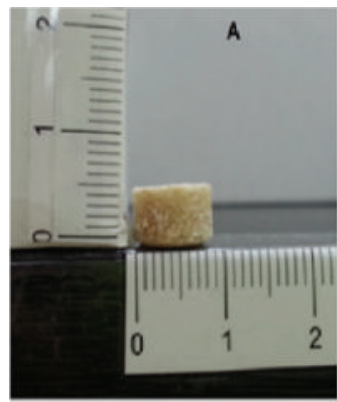

(a)

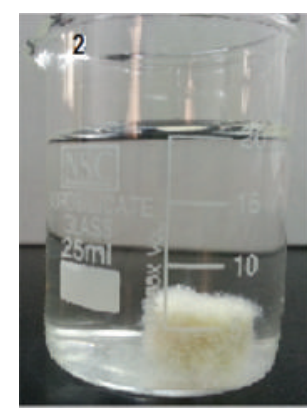

(2)

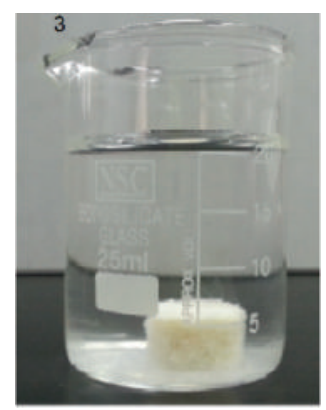

(3)

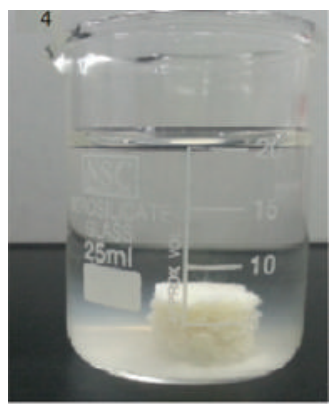

(4)

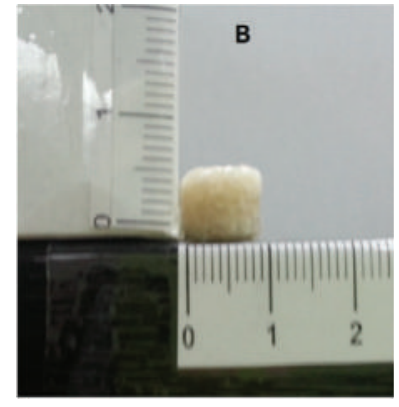

(b)

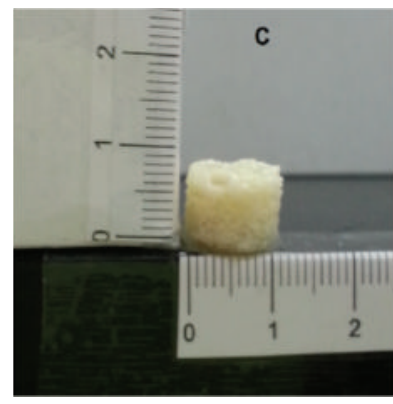

(c)

FIGURE 5: Swelling of F2 matrix tablet in pH 1.2 and 6.8 (1) at zero time, (2) after 2 hours in pH 1.2, (3) after 4 hours in pH 6.8, and (4) after 24 hours in $\mathrm{pH}$ 6.8. (a) Matrix tablet thickness and morphology at zero time, (b) after 2 hours in pH 1.2, and (c) after 24 hours in pH 6.8.

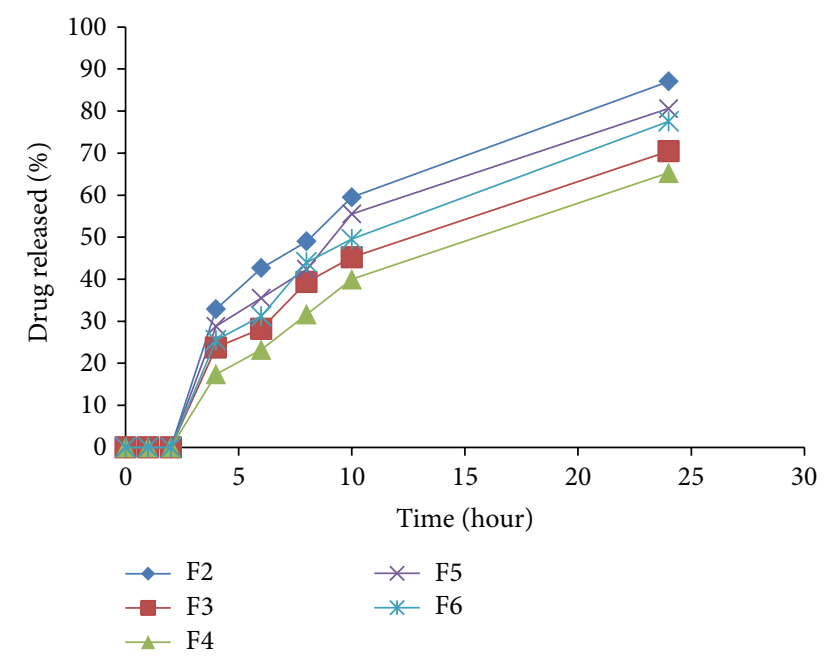

FIGURE 6: Release profiles of IDM from sustained release matrix tablets prepared from the liquisolid formulations: F2-F6.

of PEC gel network took place after 2 hours as manifested by the white cloud present in the beaker. Both intensity and magnitude of the white gelatinous cloud increased by time; the maximum erosion and dissolution were attained after 24 hours.

5.3. In Vitro Release Study. Release profiles of IDM from all formulations are presented in Figure 6. In general, IDM release from the different tablet matrices was blocked in $\mathrm{pH}$
1.2. The retardation effect of the insoluble E-RL polymer on IDM release rate from the different formulations is a common factor. The second common factor is the low $\mathrm{pKa}$ (4.5) of IDM and hence its solubility in pH 1.2.

The polymer side chain of E-RL 100 contains quaternary ammonium groups which is hydrophilic and facilitates the interaction of the polymer with water and thereby increases its permeability and allows the water to permeate the drug core in a controlled manner. The permeability and water uptake of acrylic polymers containing quaternary ammonium groups are influenced by the different counterions present in the medium. In acidic medium, polymethacrylates polymer contains a positively polarized quaternary ammonium group which is attracted by the negative hydrochloride counterions. Thus, the degree of polymer swelling and related drug release rate were functions of the chloride counterion interaction with the polymer's quaternary ammonium groups. In the presence of extra chloride ion, possibility of ion-exchange needed for hydration would be more restrictive [46]. In all tablet matrices, degree of swelling and permeability of E-RL in acidic medium could be extremely lowered or even prevented resulting in decrease in drug release rate.

The presence of PEC in F2 matrix tablet potentiated the retardation effect of E-RL on drug release. This finding could be explained by the fact that PEC contains carboxylic functional groups which are unionized, in $\mathrm{pH}$ 1.2, forming a gel network which acted as a diffusion barrier for drug release. Thus, the synergetic effect of both polymers inhibited the drug release in the acidic medium.

On immersing tablet matrix of $\mathrm{CH}$ into the acidic medium, free amino groups of $\mathrm{CH}$ got protonated and 
their hydration increased the degree of matrix swelling. Full ionization of all amino groups turned it into a polyelectrolyte with a relatively high charge density. In this system, the electrostatic repulsion of free ionized amino groups is responsible for swelling [48]. In addition, in acidic medium, the protonated carboxylic groups of E-RL (weak polyacid) become charged by ionized amino groups of $\mathrm{CH}$ to form interpolymers interaction. This polymer interaction prevented erosion of $\mathrm{CH}$ in $\mathrm{pH} 1.2$ and inhibited drug release [48].

Matrix tablet of F4 formulation also did not show drug release in acidic $\mathrm{pH}$. This is attributed to the hydration of GG by coming in contact with the dissolution media and its subsequent gel formation on the matrix tablet surface and within the matrix itself. The produced gel presented a barrier against drug molecules diffusion from the matrix in spite of the increased solubility of chitosan in acidic medium [49].

In case of tablet matrices of formulations F5 and F6 containing $\mathrm{PEC}$ and $\mathrm{CH}$ in the ratios of $3: 1$ and $1: 3$, respectively, there is no possibility of electrostatic interaction between $\mathrm{PEC}$ and $\mathrm{CH}$ as the former is present in the unionized form in acidic medium. But PEC formed a stable gel when the matrix was immersed in the acidic $\mathrm{pH}$. On the other hand, as discussed before, the ionized amino groups of $\mathrm{CH}$ interacted with the protonated carboxylic groups of E-RL. The behavior of both polymers in acidic medium prevented drug release in $\mathrm{pH}$ 1.2.

Drug release from all the tablet matrices in $\mathrm{pH} 6.8$ is shown in Figures 7, 8, 9, 10, and 11. On transferring the matrix to dissolution medium of $\mathrm{pH} 6.8$, carboxylic groups of E-RL became more ionized giving rise to an increase in the degree of swelling. On the other hand, the quaternary ammonium groups of E-RL became free from their interaction with chloride ions. The amino groups began to lose their charge and may be responsible for the increase in the hydrophobic units. As a result, the swelling slightly decreased in alkaline medium [46]. The net results denoted that permeability of the tablet matrices for dissolution medium increased, resulting in drug release in $\mathrm{pH}$ 6.8. Release data did not show rapid burst drug release in $\mathrm{PB}$ as E-RL took time for ion exchange and for the erosion of the gel formed earlier in acidic medium.

It is clear that F2 matrix exhibited the highest drug release as $32.89 \%$ of IDM was released after 2 hours of dissolution test in $\mathrm{pH}$ 6.8. However, F3, F4, F5, and F6 released 23.79, $17.41,29.88$, and $26.66 \%$ of their drug content, respectively, after the same time. After 2 hours of release experiment, an approximately linear sustained drug release occurred which could be due to the action of the time-dependent E-RL polymer and the hydration and swelling characteristics of the polysaccharide incorporated in the matrices. Highest IDM release from F2 matrix tablet was attributed to hydration and swelling of both E-RT and PEC in buffer medium. The permeability of the matrix increased and facilitated the penetration of dissolution medium to the inside of the matrix. In addition, according to FT-IR spectra (Figures 3(a) and $3(\mathrm{~b})$ ), there was no possible interaction between the polymers which could affect drug release [46].

Matrix tablet of LS formulation F3 showed a decrease in percentage of drug release rate (23.79\%) compared to F2

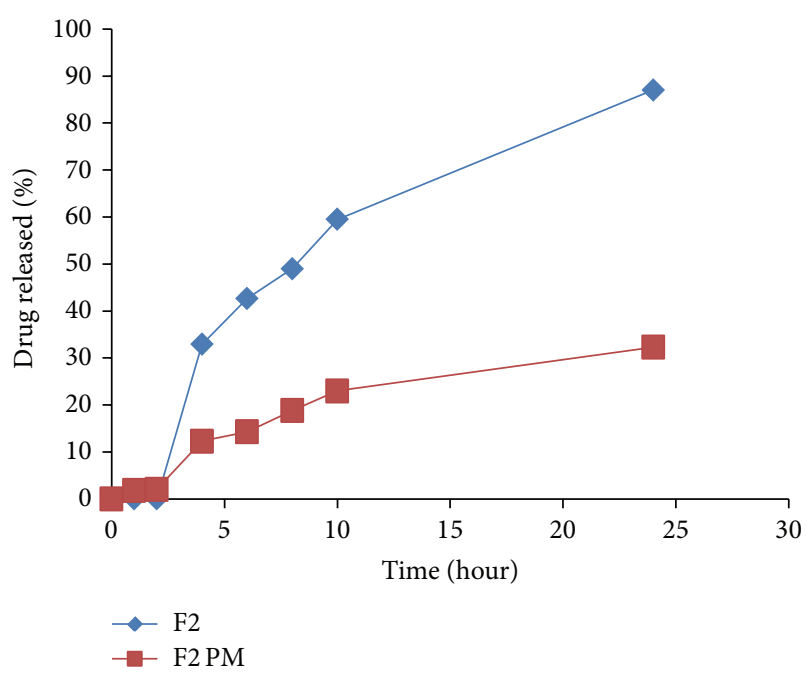

FIGURE 7: Release profiles of IDM from sustained release tablets F2 and its corresponding compressed tablet of the physical mixture F2 PM.

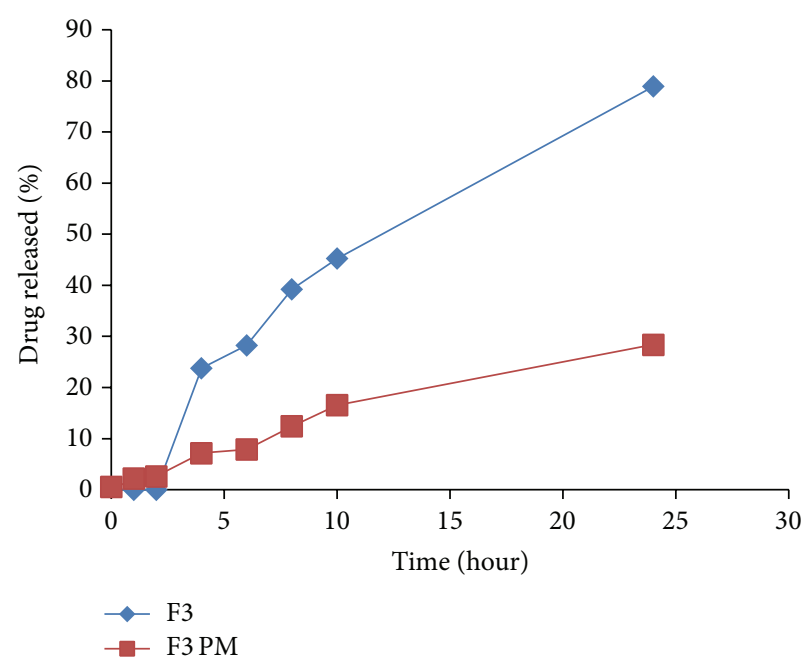

Figure 8: Release profiles of IDM from sustained release tablets F3 and its corresponding compressed tablet of the physical mixture F3 PM.

(32.89\%). This may be attributed to the decreased solubility of $\mathrm{CH}$ in higher $\mathrm{pH}$ values [48]. However, F4 matrix tablet showed the least drug release rate as about $17.41 \%$ of the drug content was released after 2 hours in $\mathrm{pH}$ 6.8. This may be attributed to the slow erosion of GG gel previously formed in acidic medium, the slight swelling of E- $\mathrm{RL}$ in $\mathrm{pH} 6.8$, and the decreased solubility of $\mathrm{CH}$ in higher pHs. In addition, it has been reported that there is a polyionic interaction between $\mathrm{CH}$ and GG molecules which retarded the hydration ability of these polymers and reduced the rate of erosion of the matrix [49].

On transferring to $\mathrm{pH} 6.8$, matrix tablet $\mathrm{F} 5$ showed higher drug release than F6. The increase in drug release rate from F5 may be explained by the inclusion of higher percentage of PEC which is soluble than chitosan in alkaline medium. 


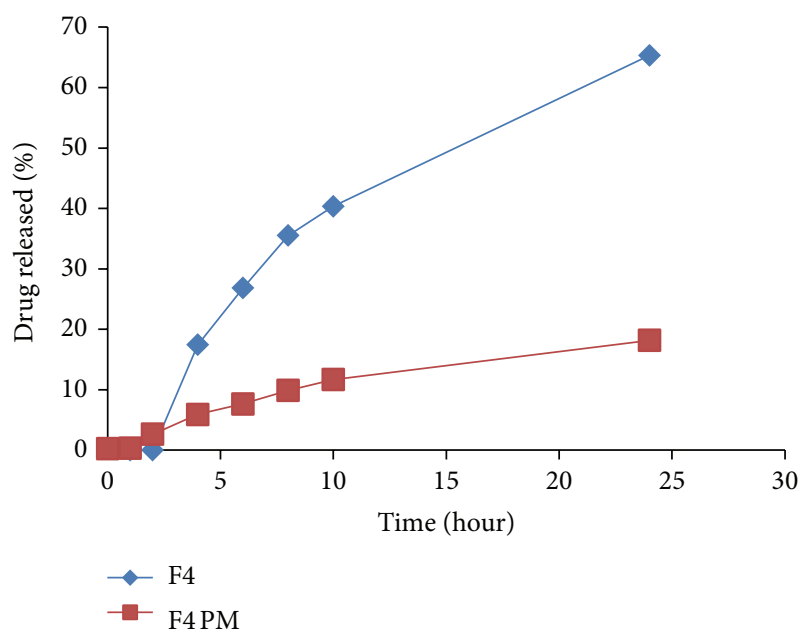

FIGURE 9: Release profiles of IDM from sustained release tablets F4 and its corresponding compressed tablet of the physical mixture F4 PM.

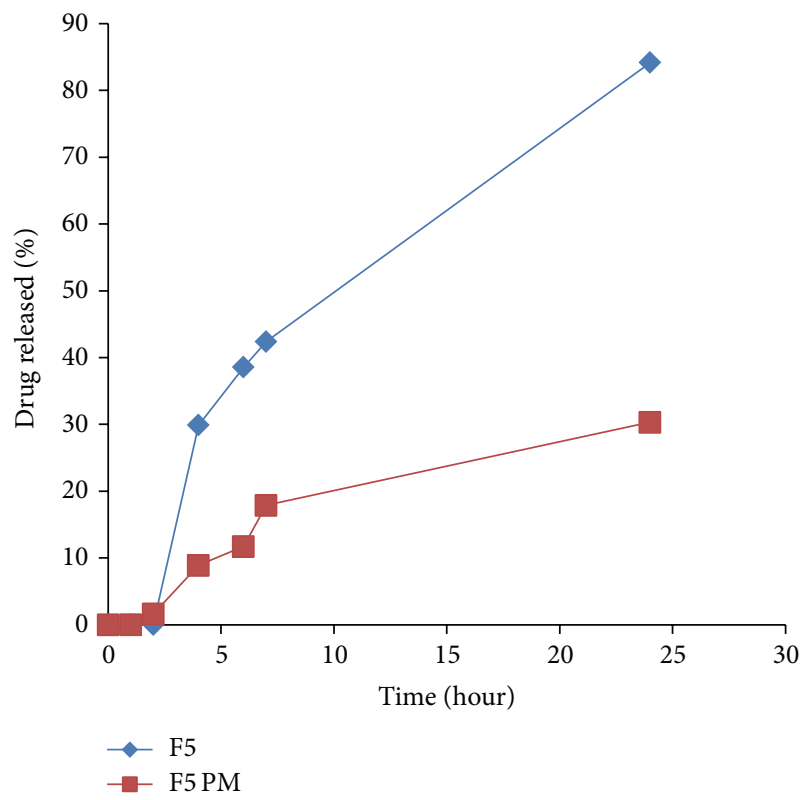

FIGURE 10: Release profiles of IDM from sustained release tablets F5 and its corresponding compressed tablet of the physical mixture F5 PM.

The release data revealed that all formulations succeeded to sustain drug release over a period of 24 hours. Percentage of drug release of $87.03,70.47,65.31,80.59$, and 77.54 was obtained from F2, F3, F4, F5, and F6 matrices, respectively. Statistical analysis of release data indicated that the difference in drug release rate was not significant $(P>0.05)$ between the five different sustained release matrix tablets.

To emphasize the efficacy of LS system to improve IDM release from controlled colon targeted matrices, tablets prepared by direct compression of the physical mixtures of all the matrix components were subjected to in vitro release studies. The release profiles are illustrated in Figures 7-11. It is obvious that the drug release from matrices containing the

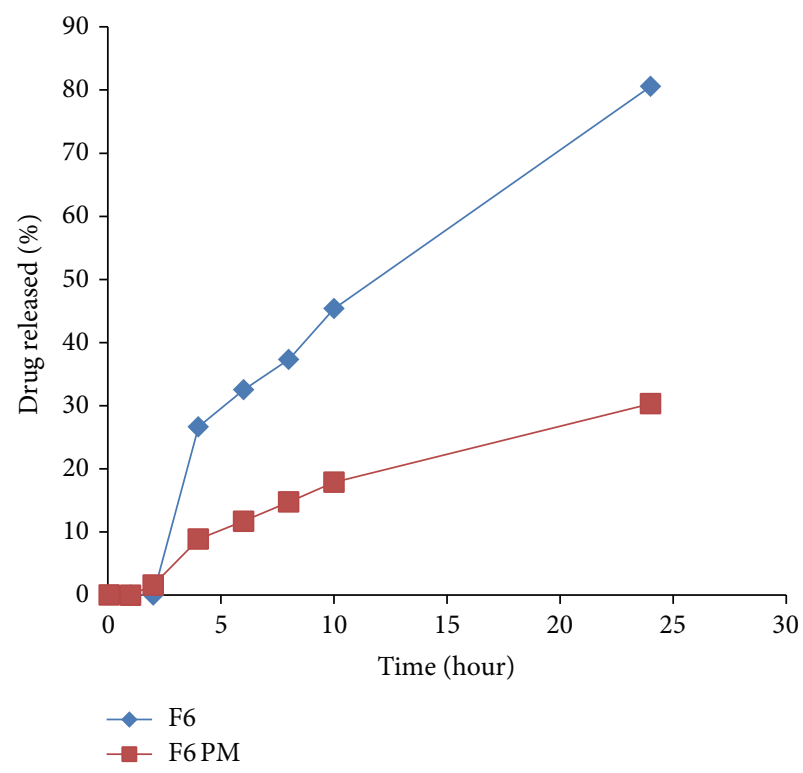

FIGURE 11: Release profiles of IDM from sustained release tablets F6 and its corresponding compressed tablet of the physical mixture F6 PM.

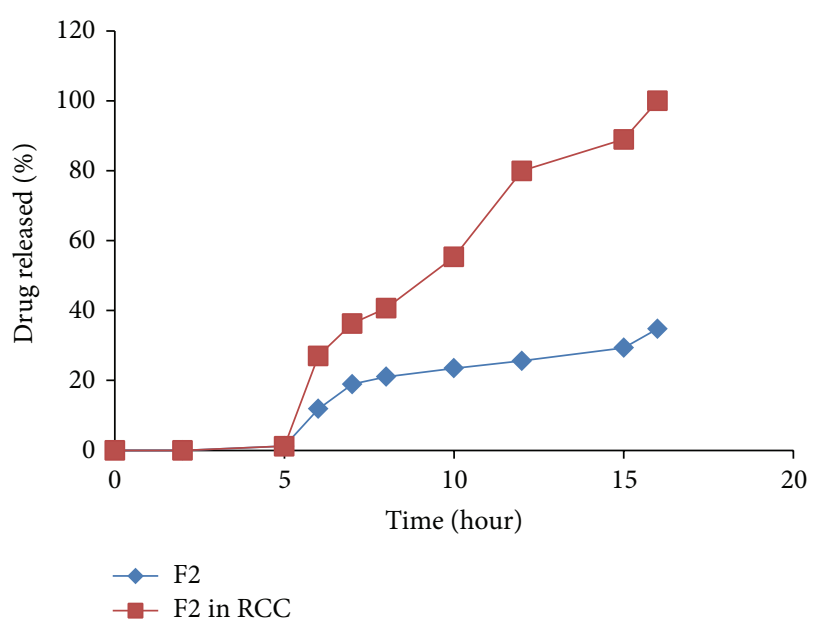

FIGURE 12: Release profiles of IDM from matrix tablet prepared from liquisolid system F2 in PB pH 6.8 and $\mathrm{PB}$ pH 6.8 containing rat cecal contents $(2 \% \mathrm{w} / \mathrm{v})$.

IDM-LS systems was much higher than that incorporating IDM powder in physical mixtures. For example, F2 matrix tablets released $42.67 \%$ of its drug content after 4 hours of release test in $\mathrm{pH}$ 6.8, whereas $\mathrm{F} 2$ conventional tablet released only $14.32 \%$ after the same time. The results of statistical analysis indicated that there was significant difference $(P<$ $0.05)$ in drug release rate between matrix tablets prepared using liquisolid systems and those prepared using physical mixture.

\section{Kinetics of Release Studies}

It was observed that the in vitro release profiles of drug from all these formulations can be best expressed by Higuchi equation as the correlation coefficients showed the higher 


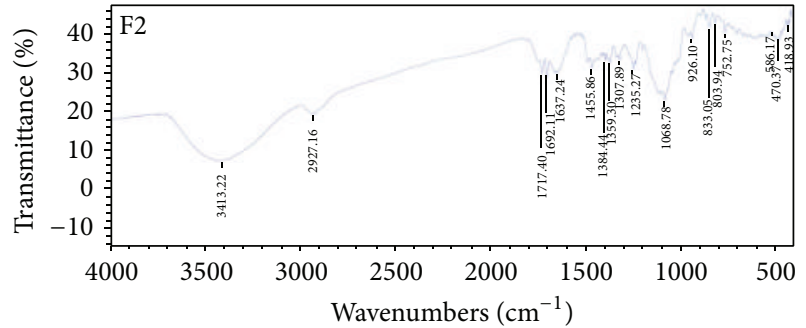

(a)

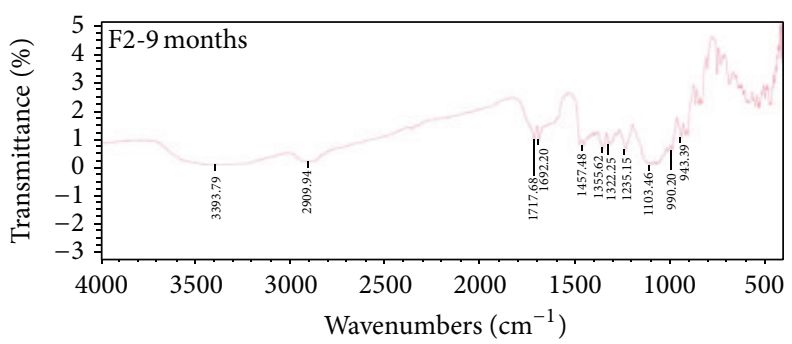

(b)

FIGURE 13: FT-IR spectra of liquisolid system (F2) before and after storage for 9 months.

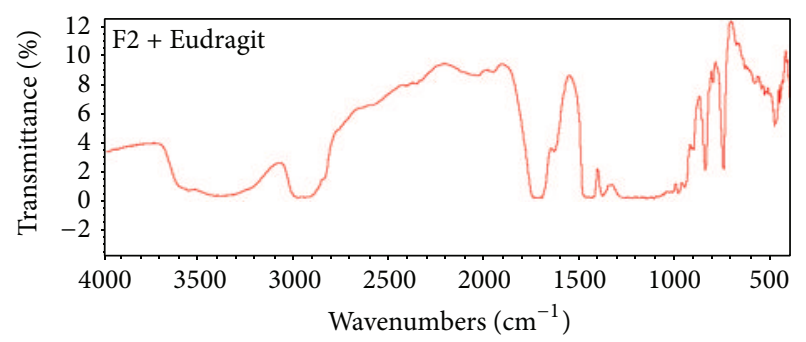

(a)

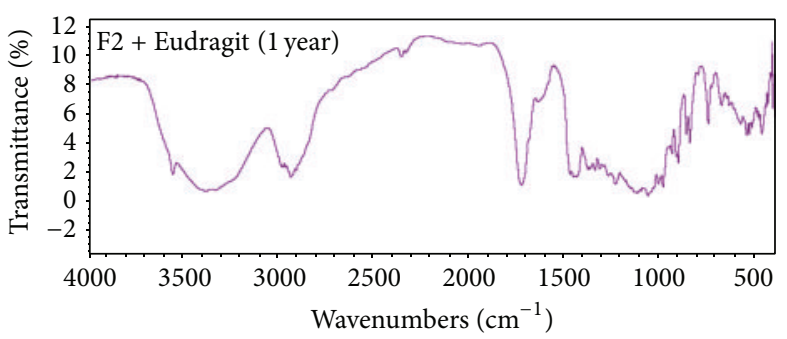

(b)

FIGURE 14: FT-IR spectra of matrix tablets (F2) before and after storage for 12 months.

values $\left(R^{2}: 0.996\right.$ for F4 to 0.993 for both F3 and F5) (Table 7). Higuchi's kinetics explains why the drug diffuses at a comparatively slower rate ( 0.053 for F2-0.060 for F3) as the distance for diffusion increases. All the formulations showed slope $(n)$ values ranging from 0.557 for F3 to 1.84 for F2. The $n$ values for formulations $\mathrm{F} 3$ indicated non-Fickian-diffusion which refers to a combination of both diffusion and erosion controlled-drug release. The other formulations showed $n$ values higher than 0.89 indicating Super Case II which refers to relaxation and erosion of the polymeric chain [28]. Release kinetics studies of compressed tablets of PMs containing IDM showed the same results. The $n$ values for formulations F14 indicated Fickian-diffusion, whereas $n$ values for all the other formulations denoted Super Case II (Table 7).

At this stage of the study, it can be concluded that F2 matrix tablet is the formulation of choice as it prevented the release of IDM in the acidic medium and permitted its release in $\mathrm{pH}$ 6.8. Therefore, F2 matrix tablet was subjected to further in vitro release study using three different release media. The ability of matrix tablets of IDM (F2) to remain intact in the physiological environment of stomach and small intestine was assessed by conducting drug release studies under conditions mimicking mouth to colon transit. Drug release studies were carried out for 2 hours in $0.1 \mathrm{~N} \mathrm{HCl}$ and then in PB pH 7.4 for 3 hours. After that, the experiment was continued up to 24 hours in $\mathrm{pH} 6.8$ [49].

The release profiles of IDM from F2 matrix tablet in absence and presence of rat cecal contents are presented in Figure 12. It was found that IDM release was inhibited in $\mathrm{pH}$ 1.2. Inhibition of drug release in acidic medium may be attributed to the insolubility and formation of the gel network of PEC as well as the decreased permeability of E-RL 100 as discussed before. On using $\mathrm{PB}$ of $\mathrm{pH}$ 7.4, the previously gelled matrix tablets, in acidic medium, decreased the rate of penetration of dissolution medium into the tablets. The outer gelled layer increased the dissolution medium diffusion coefficient and acted as barrier for drug release. The outer layer of the tablets containing E-RL associated with the chloride ions through its protonated amino groups began to dissociate liberating protonated amino groups. The protonated ammonium groups started their charge loss, producing hydrophobic units which decreased hydration and swelling of the polymer. All these factors resulted in inhibition of drug release in $\mathrm{pH}$ 7.4. Near the end of the dissolution rate experiment in $\mathrm{pH} 7.4$, it was found that about $0.90 \%$ of the drug content was released.

In $\mathrm{pH} 6.8$ without rat cecal content, F2 matrix tablet released about $19.45 \%$ of its IDM content after one hour, whereas, in presence of rat cecal contents, percentage of drug release rate increased approximately to $27.04 \%$. At the end of the 15-hour release test, it was observed that the drug release in presence of rat cecal contents was significantly increased compared to that in absence of rat cecal contents $(P<$ 0.05 ). The results of release rate studies indicated that F2 LS formulation in the form of tablets succeeded to sustain drug release over a period of 24 hours, yet it failed to comply with the USP official limits of sustained drug release in absence of rat cecal content.

Drug release rate in $\mathrm{pH} 6.8$ was below the official limits (of dissolution test 2) at the specified time intervals (USP). After 1, 2, 4, 12, and 24 hours of dissolution test, the sustained release matrix tablet of F2 released 11.28 (less than 15\%), 18.94 (less than 35\%), 22.63 (less than 55\%), 25.6 (less than 75\%), and 54.79 (less than $85 \%$ ) of its drug content, respectively. In presence of rat cecal content in a concentration of $2 \% \mathrm{w} / \mathrm{v}$, drug release rate increased to be within the official limits. 
TABLE 7: Kinetics study of drug release.

\begin{tabular}{|c|c|c|c|c|c|c|c|c|}
\hline \multirow{2}{*}{ Formula code } & \multicolumn{2}{|c|}{ Zero-order } & \multicolumn{2}{|c|}{ First-order } & \multicolumn{2}{|c|}{ Higuchi model } & \multicolumn{2}{|c|}{ Korsmeyer-Pappas' model } \\
\hline & $R^{2}$ & $k$ & $R^{2}$ & $k$ & $R^{2}$ & $k$ & $R^{2}$ & $n$ \\
\hline $\mathrm{F} 2$ & 0.919 & 0.221 & 0.980 & 0.374 & 0.995 & 0.053 & 0.996 & 1.840 \\
\hline $\mathrm{F} 2 \mathrm{PM}$ & 0.929 & 0.626 & 0.064 & 0.016 & 0.971 & 0.126 & 0.975 & 0.809 \\
\hline F3 & 0.940 & 0.285 & 0.979 & 0.423 & 0.993 & 0.060 & 0.921 & 0.557 \\
\hline F3 PM & 0.983 & 0.812 & 0.107 & 0.025 & 0.968 & 0.151 & 0.978 & 0.976 \\
\hline $\mathrm{F} 4$ & 0.962 & 0.321 & 0.982 & 0.416 & 0.996 & 0.059 & 0.990 & 1.320 \\
\hline F4 PM & 0.927 & 0.115 & 0.416 & 0.077 & 0.965 & 0.216 & 0.978 & 0.964 \\
\hline F5 & 0.931 & 0.244 & 0.974 & 0.378 & 0.989 & 0.054 & 0.990 & 0.165 \\
\hline F5 PM & 0.966 & 0.713 & 0.331 & 0.063 & 0.973 & 0.139 & 0.974 & 0.780 \\
\hline F6 & 0.940 & 0.258 & 0.977 & 0.381 & 0.993 & 0.054 & 0.989 & 1.560 \\
\hline F6 PM & 0.966 & 0.713 & 0.157 & 0.038 & 0.979 & 0.180 & 0.959 & 0.937 \\
\hline
\end{tabular}

F: matrix tablet prepared from liquisolid system.

F PM: matrix tablet prepared from physical mixture.

It was also observed that $100 \%$ drug release rate was attained within 16 hours, which is the normal residence time of a solid dosage form in the colon. Therefore, it can be concluded that F2 matrix tablet would be considered as a promising sustained release formulation of IDM for colon delivery.

\section{Stability Studies}

Shelf stability study of LS system (F2) indicated, after 3, 6,9 , and 12 months of storage at room temperature in a desiccator, no changes in the physical appearance and drug contents of either LS system or its tablet matrix. Their release profiles before and after storage revealed insignificant change in release. Figure 13 demonstrates the FT-IR spectra of both fresh and aged LS systems (stored for 9 months). However, Figure 14 illustrates the FT-IR spectra of both freshly prepared matrix tablet and matrix tablet after being stored for one year. It is obvious that there is no shift in the characteristic peaks of the drug indicating absence of any chemical interactions. The obtained results revealed that formulations were stable all over the period of study and could provide a minimum shelf life of 2 years [50].

\section{Conclusion}

Liquisolid technique was applied to develop successfully controlled colon-specific drug release systems. Eudragit RL 100 was employed as time-dependent polymer in combination of bacterial degradable polysaccharides which were used in the form of liquisolid systems loaded with the drug. Eudragit RL improved the flowability and compressibility of liquisolid systems. Drug release from all matrix tablets was characterized by negligible release in the initial phase followed by controlled release for a time period of 24 hours. Matrix tablet of pectinbased liquisolid system (F2) displayed a promising sustained release of the drug (in presence of rat cecal content) for 16 hours, which is the normal residence time of a solid dosage form in the colon. Liquisolid formulation F2 as well as its matrix tablets, when subjected to stability studies, indicated no significant change in physical appearance, drug content, and in vitro release pattern. Furthermore, no physical and chemical interaction was evident from FT-IR studies, indicating stability of indomethacin in the prepared matrices. An advantage of such a matrix design that comprises timedependent polymers in polysaccharide matrices is that it can overcome the drawbacks of coated systems wherein there is a possibility of the coat remaining insoluble during its passage through the colon.

\section{Conflict of Interests}

The authors declare that there is no conflict of interests regarding the publication of this paper.

\section{Acknowledgment}

This research project was supported by a grant from the "Research Center of the Female Scientific and Medical Colleges," Deanship of Scientific Research, King Saud University.

\section{References}

[1] M. K. Chourasia and S. K. Jain, "Pharmaceutical approaches to colon targeted drug delivery systems," Journal of Pharmacy and Pharmaceutical Sciences, vol. 6, no. 1, pp. 33-66, 2003.

[2] V. R. Sinha and R. Kumria, "Polysaccharides in colon-specific drug delivery," International Journal of Pharmaceutics, vol. 224, no. 1-2, pp. 19-38, 2001.

[3] T. Bussemer, I. Otto, and R. Bodmeier, "Pulsatile drug-delivery systems," Critical Reviews in Therapeutic Drug Carrier Systems, vol. 18, no. 5, pp. 433-458, 2001.

[4] M. S. Chavan, V. P. Sant, and M. S. Nagarsenker, "Azo-containing urethane analogues for colonic drug delivery: synthesis, characterization and in-vitro evaluation," Journal of Pharmacy and Pharmacology, vol. 53, no. 6, pp. 895-900, 2001.

[5] V. R. Vemula, V. Lagishetty, and S. Lingala, "Solubility enhancement techniques," International Journal of Pharmaceutical Sciences Review and Research, vol. 5, no. 1, pp. 41-51, 2010. 
[6] I. Sridhar, A. Doshi, B. Joshi, V. Wankhede, and J. Doshi, "Solid dispersions: an approach to enhance solubility of poorly water soluble drug," Journal of Scientific and Innovative Research, vol. 2, no. 3, pp. 685-694, 2013.

[7] N. Blagden, M. de Matas, P. T. Gavan, and P. York, "Crystal engineering of active pharmaceutical ingredients to improve solubility and dissolution rates," Advanced Drug Delivery Reviews, vol. 59, no. 7, pp. 617-630, 2007.

[8] S. Anjumn, A. Farhan, P. Yvonne, and R. M. Afzal, "Effects of ball-milling on PLGA polymer and its implication on lansoprazole-loaded nanoparticles," Journal of Basic and Clinical Pharmacy, vol. 2, no. 2, pp. 71-81, 2011.

[9] M. L. Hart, D. P. Do, R. A. Ansari, and S. A. A. Rizvi, "Brief overview of various approaches to enhance drug solubility," Journal of Developing Drugs, vol. 2, no. 3, Article ID 100011, 2013.

[10] J. Reza, "Self-emulsifying drug delivery systems: a review," International Journal of Pharmaceutical and Life Sciences, vol. 2, no. 2, pp. 80-84, 2013.

[11] N. Chella, N. Shastri, and R. R. Tadikonda, "Use of the liquisolid compact technique for improvement of the dissolution rate of valsartan," Acta Pharmaceutica Sinica B, vol. 2, pp. 502-508, 2012.

[12] S. Spireas and S. M. Bolton, "Liquisolid systems and method of preparing same," U.S Patent 6096337, 2000.

[13] S. Spireas, Liquisolid System and method of preparing same, U.S Patent 6423339 B1, 2002.

[14] P. Chandel, K. Raj, and A. Kapoor, "Liquisolid technique: an approach for enhancement of solubility," Journal of Drug Delivery \& Therapeutics, vol. 3, no. 4, pp. 131-137, 2013.

[15] S. S. Spireas, C. I. Jarowski, and B. D. Rohera, "Powdered solution technology: principles and mechanism," Pharmaceutical Research, vol. 9, no. 10, pp. 1351-1358, 1992.

[16] Y. Javadzadeh, M. R. Siahi-Shadbad, M. Barzegar-Jalali, and A. Nokhodchi, "Enhancement of dissolution rate of piroxicam using liquisolid compacts," Farmaco, vol. 60, no. 4, pp. 361-365, 2005.

[17] A. Nokhodchi, C. M. Hentzschel, and C. S. Leopold, "Drug release from liquisolid systems: speed it up, slow it down," Expert Opinion on Drug Delivery, vol. 8, no. 2, pp. 191-205, 2011.

[18] B. M. El-Houssieny, L. F. Wahman, and N. M. S. Arafa, "Bioavailability and biological activity of liquisolid compact formula of repaglinide and its effect on glucose tolerance in rabbits," Bioscience Trends, vol. 4, no. 1, pp. 17-24, 2010.

[19] Y. Javadzadeh, B. Jafari-Navimipour, and A. Nokhodchi, "Liquisolid technique for dissolution rate enhancement of a high dose water-insoluble drug (carbamazepine)," International Journal of Pharmaceutics, vol. 341, no. 1-2, pp. 26-34, 2007.

[20] K. A. Khaled, Y. A. Asiri, and Y. M. El-Sayed, "In vivo evaluation of hydrochlorothiazide liquisolid tablets in beagle dogs," International Journal of Pharmaceutics, vol. 222, no. 1, pp. $1-6,2001$.

[21] M. D. Boudreau, K. H. Sohn, S. H. Rhee, S. W. Lee, J. D. Hunt, and D. H. Hwang, "Suppression of tumor cell growth both in nude mice and in culture by n-3 polyunsaturated fatty acids: mediation through cyclooxygenase-independent pathways," Cancer Research, vol. 61, no. 4, pp. 1386-1391, 2001.

[22] D. J. E. Elder, D. E. Halton, T. E. Crew, and C. Paraskeva, "Apoptosis induction and cyclooxygenase-2 regulation in human colorectal adenoma and carcinoma cell lines by the cyclooxygenase-2-selective non- steroidal anti-inflammatory drug NS-398," International Journal of Cancer, vol. 86, no. 4, pp. 553-560, 2000.
[23] C. Lönnroth, M. Andersson, and K. Lundholm, "Indomethacin and telomerase activity in tumor growth retardation," International Journal of Oncology, vol. 18, no. 5, pp. 929-937, 2001.

[24] K. Higuchi, E. Umegaki, T. Watanabe et al., "Present status and strategy of NSAIDs-induced small bowel injury," Journal of Gastroenterology, vol. 44, no. 9, pp. 879-888, 2009.

[25] T. Higuchi and K. A. Connors, "Phase solubility techniques," Advances in Analytical Chemistry and Instrumentation, vol. 4, pp. 117-212, 1965.

[26] S. Spireas and S. M. Bolton, "Liquisolid systems and methods of preparing same," U.S. Patent 5968550, 1999.

[27] P. E. Luner, L. E. Kirsch, S. Majuru et al., "Preformulation studies on the S-isomer of oxybutynin hydrochloride, an Improved Chemical Entity (ICEUं)," Drug Development and Industrial Pharmacy, vol. 27, no. 4, pp. 321-329, 2001.

[28] K. A. Khan, "The concept of dissolution efficiency," Journal of Pharmacy and Pharmacology, vol. 27, no. 1, pp. 48-49, 1975.

[29] Y. S. R. Krishnaiah, P. R. Bhaskar Reddy, V. Satyanarayana, and R. S. Karthikeyan, "Studies on the development of oral colon targeted drug delivery systems for metronidazole in the treatment of amoebiasis," International Journal of Pharmaceutics, vol. 236, no. 1-2, pp. 43-55, 2002.

[30] Y. V. R. Prasad, Y. S. R. Krishnaiah, and S. Satyanarayana, "In vitro evaluation of guar gum as a carder for colon-specific drug delivery," Journal of Controlled Release, vol. 51, no. 2-3, pp. 281287, 1998.

[31] R. W. Korsmeyer, R. Gurny, and E. Doelker, "Mechanisms of solute release from porous hydrophilic polymers," International Journal of Pharmaceutics, vol. 15, no. 1, pp. 25-35, 1983.

[32] A. Nokhodchi, Y. Javadzadeh, M. R. Siahi-Shadbad, and M. Barzegar-Jalali, "The effect of type and concentration of vehicles on the dissolution rate of a poorly soluble drug (indomethacin) from liquisolid compacts," Journal of Pharmacy and Pharmaceutical Sciences, vol. 8, no. 1, pp. 18-25, 2005.

[33] S. Basavoju, D. Boström, and S. P. Velaga, "Indomethacinsaccharin cocrystal: design, synthesis and preliminary pharmaceutical characterization," Pharmaceutical Research, vol. 25, no. 3, pp. 530-541, 2008.

[34] D. G. Oakenfull, "The chemistry of high-methoxyl pectins," in The Chemistry and Technology of Pectin, R. H. Walter, Ed., Academic Press, New York, NY, USA, 1991.

[35] USP 29- NF 24, Page 3017.

[36] S. I. Ahmed, S. J. Mohan, and Y. M. Rao, "Modulating the release behavior and kinetic evaluation of diclofenac sodium from natural polymers," International Journal of ChemTech Research, vol. 2, no. 2, pp. 834-841, 2010.

[37] P. Apparao, J. V. Prabhakarreddy, J. Raju, and B. Shashidher, "Formulation and evaluation of gum based matrix tablets of Lamivudine," Der Pharmacia Sinica, vol. 2, no. 3, pp. 176-192, 2011.

[38] A. A. S. Sundar Raj, S. Rubila, R. Jayabalan, and T. V. Ranganathan, "A Review on Pectin: Chemistry due to General Properties of Pectin and its Pharmaceutical Uses," http://www .omicsonline.org/scientific-reports/srep550.php.

[39] M. Md. Islama, S. Md. Masumb, M. M. Rahmana, A. I. Md. Mollab, A. A. Shaikhc, and S. K. Roy, "Preparation of chitosan from shrimp shell and investigation of its properties," International Journal of Basic \& Applied Sciences, vol. 11, no. 1, pp. 77-80, 2011.

[40] M. Chaplin, Water Structure and Behavior: Guar Gum, South Bank University, London, UK, 2006. 
[41] S. S. Kadian and S. L. Harikumar, "Eudragit and its pharmaceutical significance. PharmainfoNet," 2009, http://www.pharmainfo.net/satishsinghkadian/publications/eudragit-and-its.

[42] A. Paharia, A. K. Yadav, G. Rai, S. K. Jain, S. S. Pancholi, and G. P. Agrawal, "Eudragit-coated pectin microspheres of 5fluorouracil for colon targeting," AAPS PharmSciTech, vol. 8, no. 1, article 12, 2007.

[43] B. Nath, L. Nath, and P. Kumar, "Preparation and in vitro dissolution profile of zidovudine loaded microspheres made of Eudragit RS 100, RL 100 and their combinations," Acta Poloniae Pharmaceutica-Drug Research, vol. 68, no. 3, pp. 409-415, 2011.

[44] R. Cortesi, L. Ravani, E. Menegatti, and E. Esposito F Ronconi, "Eudragit microparticles for the release of budesonide: a comparative study," Indian Journal of Pharmaceutical Sciences, vol. 74, no. 5, pp. 415-421, 2012.

[45] A. Akhgari, M. Abbaspour, and M. Moradkhanizadeh, "Combination of pectin and Eudragit RS and Eudragit RL in the matrix of pellets prepared by extrusion-spheronization for possible colonic delivery of 5-aminosalicylic acid," Jundishapur Journal of Natural Pharmaceutical Products, vol. 8, no. 2, pp. 86-92, 2013.

[46] A. Akhgari, H. Afrasiabi Garekani, and F. Sadeghi, "Combination of inulin and time dependent polymethacrylates as a coating system to achieve colonic delivery of indomethacin," Daru, vol. 17, no. 3, pp. 199-208, 2009.

[47] M. Efentakis and M. Vlachou, "Evaluation of high molecular weight poly(oxyethylene) (Polyox)polymer: studies of flow properties and release rates of furosemide and captopril from controlled-release hard gelatin capsules," Pharmaceutical Development and Technology, vol. 5, no. 3, pp. 339-346, 2000.

[48] K. C. Ofokansi and F. C. Kenechukwu, "Formulation development and evaluation of drug release kinetics from colontargeted ibuprofen tablets based on eudragit RL 100-chitosan inter-polyelectrolyte complexes," ISRN Pharmaceutics, vol. 2013, Article ID 838403, 8 pages, 2013.

[49] S. J. Shankar, S. B. Gaurav, and B. V. Basavaraj, "Formulation and evaluation of controlled release matrix tablets of an antimicrobial drug," International Journal of Pharmaceutical Research and Development, vol. 2, no. 10, pp. 8-10, 2010.

[50] B. R. Matthews, "Regulatory aspects of stability testing in Europe," Drug Development and Industrial Pharmacy, vol. 25, no. 7, pp. 831-856, 1999. 

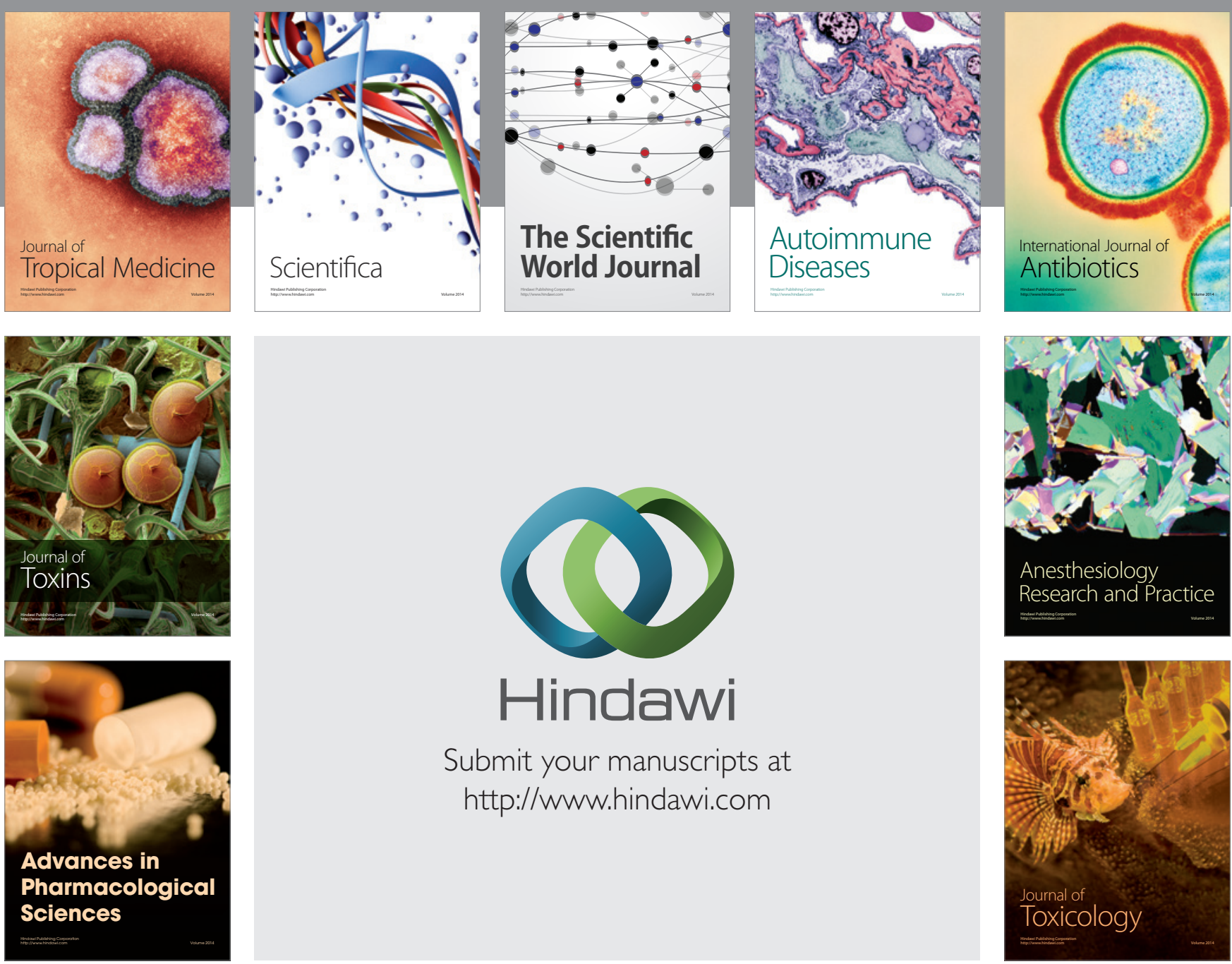

\section{Hindawi}

Submit your manuscripts at

http://www.hindawi.com
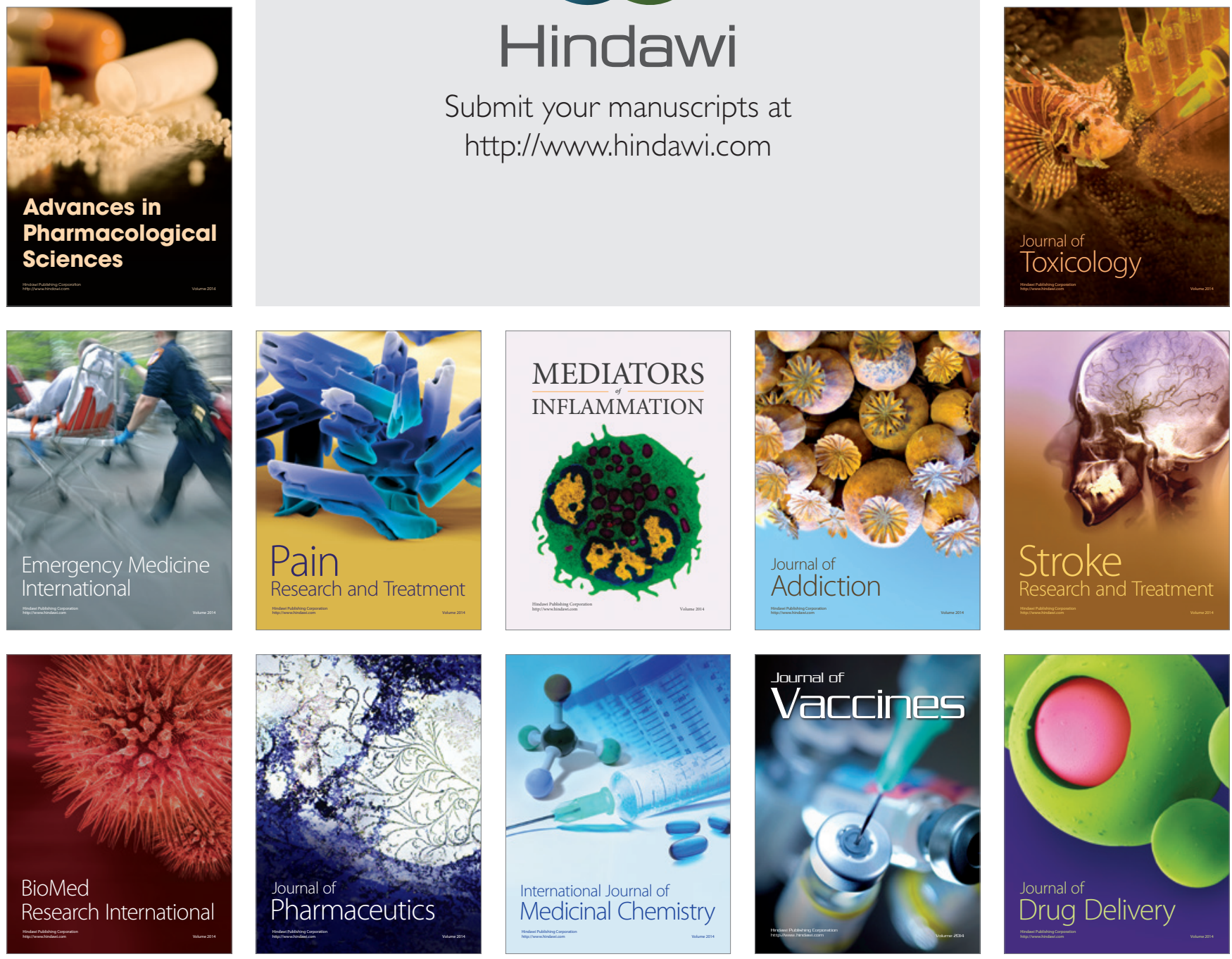